This item was submitted to Loughborough's Research Repository by the author.

Items in Figshare are protected by copyright, with all rights reserved, unless otherwise indicated.

\title{
Characterisation of high 1,3-distearoyl-2-oleoyl-sn-glycerol content stearins produced by acidolysis of high oleic sunflower oil with stearic and palmitic acids
}

\section{PLEASE CITE THE PUBLISHED VERSION}

http://dx.doi.org/10.1002/ejlt.201300282

\section{PUBLISHER}

(C) WILEY-VCH Verlag $\mathrm{GmbH}$ and Co

\section{VERSION}

AM (Accepted Manuscript)

\section{PUBLISHER STATEMENT}

This work is made available according to the conditions of the Creative Commons Attribution-NonCommercialNoDerivatives 4.0 International (CC BY-NC-ND 4.0) licence. Full details of this licence are available at: https://creativecommons.org/licenses/by-nc-nd/4.0/

\section{LICENCE}

CC BY-NC-ND 4.0

\section{REPOSITORY RECORD}

Ray, Joydeep, Kevin W. Smith, Krishnadath Bhaggan, Zoltan K. Nagy, and A.G.F. Stapley. 2014.

"Characterisation of High 1,3-distearoyl-2-oleoyl-sn-glycerol Content Stearins Produced by Acidolysis of High Oleic Sunflower Oil with Stearic and Palmitic Acids". figshare. https://hdl.handle.net/2134/16146. 


\section{Characterisation of high 1,3-Distearoyl-2-oleoyl-sn-glycerol content stearins produced by acidolysis of high oleic sunflower oil with stearic and palmitic acids}

Joydeep Ray ${ }^{1}$, Kevin W. Smith ${ }^{2}$, Krishnadath Bhaggan ${ }^{3}$, Zoltan K. Nagy ${ }^{1,4}$, Andrew G. F. Stapley ${ }^{*, 1}$

${ }^{1}$ Department of Chemical Engineering, Loughborough University, Loughborough, Leicestershire, LE11 3TU, UK.

${ }^{2}$ Fat Science Consulting Ltd, 16 Arundel Drive, Bedford, MK41 8HP, UK.

${ }^{3}$ IOI Loders Croklaan Europe, Hogeweg 1, 1521 AZ Wormerveer, The Netherlands.

${ }^{4}$ School of Chemical Engineering, Purdue University, West Lafayette, IN 47907-2100, USA.

\section{Running title:}

Characterisation of high StOSt hard stearin fractions

\section{${ }^{*}$ Corresponding author address:}

Department of Chemical Engineering, Loughborough University, Loughborough, Leicestershire, LE11 3TU, UK.

Phone: $+44-1509-222525$

Fax: +44-1509-223923

E-mail: A.G.F.Stapley@lboro.ac.uk

\section{Keywords:}

Diacylglycerols, Triacylglycerols, Stop-and-return DSC, Confocal Raman IR Microscopy, silica treatment, StOSt, SOS.

Publisher version available at http://www.interscience.wiley.com/

Ray, J., Smith, K. W., Bhaggan, K., Nagy, Z. K., \& Stapley, A. G. F. (2014). Characterisation of high 1,3-distearoyl-2-oleoyl-sn-glycerol content stearins produced by acidolysis of high oleic sunflower oil with stearic and palmitic acids. European Journal of Lipid Science and Technology, 116(5), 532-547. doi: 10.1002/ejlt.201300282. 

Abbreviations:
A
Arachidic acid
B
Behenic acid
CB
Cocoa Butter
CBE Cocoa Butter Equivalent
HOSO High Oleic Sunflower Oil
HRGC High Resolution Gas Chromatography
L L Linoleic acid
O Oleic acid
OX Oxidised glycerides
P Palmitic acid
P98 98\% pure palmitic acid
PLM Polarised Light Microscopy
PMF Palm Mid Fraction
POP 1,3-dipalmitoyl-2-oleoyl-sn-glycerol
POSt rac-1-palmitoyl-2-oleoyl-3-stearoyl glycerol
S95 $95 \%$ pure stearic acid
S98 $98 \%$ pure stearic acid
Sat Saturated fatty acid
Sat-O-Sat Symmetric disaturated monounsaturated
SFC Solid Fat Content
Si Silica
St Stearic acid
StOSt 1,3-distearoyl-2-oleoyl-sn-glycerol 


\section{Summary}

Nine different stearin fractions with 1,3-distearoyl-2-oleoyl-sn-glycerol (StOSt) contents ranging from $69-84 \%$ were obtained via fractionation from fats produced by acidolysis of high oleic sunflower oil (HOSO) with various mixtures of stearic (either $95 \%$ or $98 \%$ pure) and palmitic acids (98\% pure). Samples were further treated with silica to reduce the oxidised glyceride and DAG content. Isothermal crystallisation at $20^{\circ} \mathrm{C}$ showed a single main peak, but evidence of crystallisation during the initial DSC transient was also apparent for high StOSt content samples. This was confirmed as the $\alpha$ form by stop-and-return DSC and XRD. The main crystallisation event was generally faster (including a shorter induction time) for samples with higher StOSt levels (lower POSt levels). Silica treatment generally accelerated transformations to higher polymorphs $\left(\gamma, \beta^{\prime}\right.$ and $\beta$ ). Raman IR microscopy experiments showed that crystallisation of the $\beta$-form was achieved after 7 days storage at $20^{\circ} \mathrm{C}$ but only in the silica treated stearin samples. This is consistent with higher SFC values that were obtained with silica treated samples, which also increased with higher levels of StOSt. The results suggest that such stearins could potentially replace shea stearin in CBE formulations.

\section{Practical applications}

This paper has two main practical applications: 1) Provides knowledge and understanding of the crystallisation and polymorphic behaviour of isolated hard stearins, obtained from enzymatic acidolysis of high oleic sunflower oil, which can be further used in CBE formulations. 2) The presence of minor components significantly delays the crystallisation of the stearins, confirming the desirability of higher quality (less oxidised/hydrolysed) fat ingredients. 


\section{Introduction}

Cocoa Butter ( $C B$ ) is one of the most expensive and widely used raw materials in the chocolate and confectionery industries due to its unique melting characteristics and physical properties. $\mathrm{CB}$ is composed mainly of triacylglycerols (TAGs), three of which account for over $75 \%$ of the TAG composition: rac-1-palmitoyl-2-oleoyl-3-stearoyl-sn-glycerol (POSt), 1,3-disteroyl-2-oleoyl-sn-glycerol (StOSt) and 1,3 diapalmitoyl-2-oleoyl-sn-glycerol (POP), where $\mathrm{P}, \mathrm{O}$ and St stand for palmitic, oleic and stearic acid respectively [1]. These TAGs majorly dictate the physical behaviour of $\mathrm{CB}$, but other minor components e.g. diacylglycerols (DAGs) and trisaturated TAGs can also influence the melting and crystallisation behaviour [2]. Cocoa Butters of different geographical origin have been shown to have different chemical compositions and hence different physical properties $[3,4]$. Due to the rise in the cost of $C B$ in recent years, the variability in quality and the uncertainty in supply compared to other fats, the development of speciality fats with similar TAG compositions to CB and fully compatible with the physical and sensory properties of CB has been encouraged.

Alternatives to CB are mainly of three types: (a) lauric substitutes or cocoa butter substitutes (CBS), (b) non-lauric substitutes or cocoa butter replacers (CBR) and (c) cocoa butter equivalents (CBE) [5]. CBEs have a similar melting profile, molecular composition and polymorphism and are completely compatible with $\mathrm{CB}$ without presenting any eutectic behaviour [5]. According to Directive 2000/36/EC of the European Parliament and the Council, up to $5 \%$ of finished chocolate products can be substituted by vegetable fats/CBE, without reducing the minimum content of $\mathrm{CB}$ or total dry cocoa solids. However, for countries outside the EU, regulations for the usage of CBEs in chocolates vary. Generally, the hard stearin fraction of fats, high in StOSt content can be used alone or blended together with fats such as palm mid fraction (PMF) to produce CBEs. Six vegetable oils which are rich in symmetric disaturated monounsaturated TAGs (Sat-O-Sat) are currently permitted to be used as CBEs in the EU: illipe (rich in POSt and StOSt), kokum (StOSt), shea butter (StOSt), mango kernel (POSt and StOSt), sal (StOSt) and palm mid fraction (POP) [6].

Shea butter is the most popular commercial source of Sat-O-Sat and is obtained from the tree Butyrospermum parkii. Shea butter is fractionated to obtain shea stearin, which is a high melting fraction enriched in StOSt and is mainly used to produce CBEs. The availability of shea stearin depends on the supply of shea nuts which can change considerably from time to time [7]. In this regard, there is a constant demand of fat fractions with Sat-O-Sat similar in composition to that of shea stearin. There is thus an interest to develop processes to produce Sat-O-Sat rich fats from reliable and readily available cheap starting materials. For 
the past few decades, several patents have been published demonstrating different methods for the production of hard butter fat compositions to use in confectionery products from various liquid oils and fats [8-13]. Popular methods include the enzymatic interesterification or acidolysis and fractionation of relatively inexpensive oils such as palm mid fraction, palm stearin and Chinese tallow. Recently, Salas et al. [14] reported the production of different stearate-rich butter fractions by solvent fractionation of high stearic-high oleic sunflower oil which was further blended with palm mid fraction [15]. The isothermal crystallisation kinetics of the sunflower hard stearins when compared alone and with formulated CBE blends demonstrated varying crystallisation mechanisms according to their TAG composition [16].

High Oleic Sunflower Oil (HOSO) contains high levels of oleic acid ( 80\%), mainly in the form of triolein (OOO) $(>70 \%)$. It has a very low solids content at room temperature but can be structurally modified by enzymatic acidolysis to increase the overall Sat-O-Sat level. The acidolysed fats can be further fractionated to concentrate the Sat-O-Sat (as a stearin fraction) which can be used to formulate CBEs. In previous work [17], acidolysis of HOSO was carried out with 11 different stearic-palmitic acid mixtures, where stearic acids of either $95 \%$ (S95) or $98 \%$ (S98) purity were blended in different ratios with $98 \%$ purity palmitic acid (P98), at an oil: acid ratio of 1:1.3 (w/w) using a 1-3 regiospecific lipase Rhizopus oryzae. The ratio of St:P in the acidolysis reactions was varied from 98:02 to 80:20 to explore the effect of stearic acid content. New fat compositions with a high content ( 40\%) of Sat-O-Sat TAGs and with varying amounts of StOSt, POSt and POP were obtained as end products. The trialling of two different purity grades of stearic acid has commercial importance given the different costs of these grades. In addition, significant amounts of DAGs (8-10\%) with oxidised glycerides (OX) were identified in the samples as by-products of the acidolysis reactions. These minor components can be retained in the fractionated stearin fractions and highly influence the crystallisation behaviour of the stearins themselves as well as subsequent formulations of CBEs and confectionery products.

Several studies have been conducted to investigate the influence of minor components particularly DAGs, on fat crystallisation. The nature of the TAGs (chain length and saturation/unsaturation characteristics) can highly affect the crystallisation behaviour and the interaction of these TAGs with each other. Lately, Smith et al. [18] have extensively documented the influence of minor components and additives on fat crystallisation. DAGs have been found to either promote or delay crystallisation, depending on the TAG composition, the fatty acid composition of the DAG and the concentration. For palm oil, the presence of DAGs (mainly dipalmitin) has been reported to inhibit or initiate nucleation as well as retard or enhance crystal growth rates [19, 20]. A low (1\%) level prolonged the induction period and reduced crystal growth whereas a higher $(5 \%)$ level speeded up 
nucleation and crystal growth. For shea stearin the removal of DAGs by silica (Si-) treatment resulted in a shorter induction time of crystallisation and faster crystal growth [21]. The influence of DAGs has also been investigated on milk fat, rapeseed oil, cocoa butter and CBE crystallisation [22-27]. DAGs exerted different effects on the crystallisation behaviour and changes in crystal growth mechanism depending on composition, concentration, degrees of supercooling and the isomer type. An interesting finding is that 1,2 DAGs tend to hinder crystallisation and transformation [23] by poisoning the crystals (as they most resemble the TAG conformation), whereas 1,3 DAGs tend to aid nucleation due to their high melting points [25] but are less likely to poison.

The present work involves the characterisation of the hard stearins obtained via enzymic acidolysis of HOSO with several mixtures of stearic and palmitic acid previously obtained [17]. This was achieved through DSC melting, isothermal crystallisation and stop-and-return experiments, variable temperature powder XRD, Solid Fat Content (SFC) profiles via pulsed NMR, Polarised Light Microscopy (PLM) and Confocal Raman microscopy. The same techniques were also used to compare the behaviour with stearin samples that were pretreated with silica to remove polar components (mainly DAGs and oxidised glycerides). The TAG and DAG compositions of the stearins used in this study were measured by using a combination of HRGC and HPLC techniques. The characterisation of the crystallisation and polymorphic behaviour of these stearins will eventually help in the formulation of CBEs, as well as in assessing the effect of minor components.

\section{Materials and Method}

\subsection{Materials}

Stearin samples were produced by enzymatic acidolysis of HOSO with 9 different stearicpalmitic acid mixtures in batch operations, followed by short path distillation to remove residual fatty acids to below $1 \%$, as described by Ray et al. [17]. This produced fats containing an average of $40 \%$ of Sat-O-Sat TAGs. TAG standards (POSt, StOSt, and POP; $\geq 99 \%$ pure) were kindly provided by Unilever Research Colworth (Sharnbrook, U.K.). Cocoa Butter Reference Material (IRMM-801) was purchased from the Institute for Reference Materials and Measurements (Belgium). HPLC grade iso-octane and HPLC grade acetone were purchased from Sigma-Aldrich (Dorset, U.K.) and Fisher Scientific (Loughborough, U.K.) respectively. HPLC grade hexane, toluene, ethyl acetate and formic acid were purchased from Merck (Amsterdam, The Netherlands). 12-hydroxystearylalcohol was purchased from Sigma-Aldrich (Zwijndrecht, The Netherlands). 


\subsection{Isolation of stearin fractions}

Acidolysed fat samples were mixed at an oil: acetone ratio of 1:5 (w/v) and kept in the temperature range of $10-11^{\circ} \mathrm{C}$ for about $1 \mathrm{hr}$. Distilled water $(0.7 \mathrm{wt} \%)$ was also added to the mixture. The stearin crystals precipitated out and were filtered using a vacuum filtration unit. The stearin fractions were washed 2-3 times with acetone and maintained at $10-11^{\circ} \mathrm{C}$ in order to reduce the olein content in the sample. The yield of each stearin fraction recovered via fractionation of the acidolysed fat samples is expressed as:

$$
\text { Yield of stearin }(\%)=\frac{M_{S T}}{M_{E I E}} \times 100
$$

where, $M_{S T}$ is the final stearin mass and $M_{E I E}$ is the sample mass before fractionation.

\subsection{Compositional analyses by HPLC and HRGC}

Quantification of DAG content was performed following the method previously reported by Ray et al. [21] using a Waters 2695 Separations Module (Waters, Millford, USA) equipped with a Ternair HPLC pump system, an autosampler and an Alltech 500 ELSD detector (low gain) drift-tube. DAGs were separated using an Altech Econosphere Silica column of 4.6 $\mathrm{mm}$ internal diameter $\times 150 \mathrm{~mm}$ length, preloaded with silica particles of size $5 \mu \mathrm{m}$.

Determination of individual DAGs and TAGs was carried out by HRGC using the methods previously described by Ray et al. [21] using an Agilent 7890A gas chromatography system (Agilent Technologies, Edinburgh, UK) equipped with an auto-sampler, split injector and flame ionisation detector (FID). A 65\% diphenyl/35\% dimethyl polysiloxane RTx-65TG column of $30 \mathrm{~m}$ length, 0.25 internal diameter and $0.10 \mu \mathrm{m}$ film thickness (Restek, Bellefonte, USA) was used.

\subsection{Silica treatment}

To remove DAG and polar compounds, the solid stearins were first dissolved in hexane (1:4 $\mathrm{w} / \mathrm{v}$ ) and about 1:3 w/w (silica:fat) of silica gel-60 was added and mixed thoroughly for 5-10 min. The silica gel-60 was then removed via vacuum filtration and the filter cake was washed using the same quantity of hexane previously used. Finally, hexane was evaporated using a 
rotary evaporator from the combined filtrate and wash to recover the Si-treated stearin samples.

\subsection{DSC analyses}

i) Isothermal crystallisation and determination of Avrami constants: All DSC experiments were analysed using a TA Q10 DSC with a refrigerated cooling system (TA instruments, Crawley, UK). $10 \pm 1 \mathrm{mg}$ of sample was weighed and hermetically sealed in an aluminium pan, with an empty pan as a reference. The isothermal DSC method consisted of first erasing previous crystal memory by holding at $80{ }^{\circ} \mathrm{C}$ for $5 \mathrm{~min}$, followed by cooling at $25^{\circ} \mathrm{C} / \mathrm{min}$ to $20^{\circ} \mathrm{C}$ where they were held until crystallisation appeared complete, after which they were heated at $10^{\circ} \mathrm{C} / \mathrm{min}$ to $80^{\circ} \mathrm{C}$ to view the melting profiles. Integration and peak temperature measurements were performed using the manufacturer's software (Universal Analysis V4.7A, TA Instruments, Crawley, UK) using linear baselines.

The isothermal crystallisation kinetics of the stearin samples were assessed by the Avrami equation [28] supplemented by an induction time,

$$
\left(1-x_{\text {solid }}\right)=\exp \left\{-k\left(t-t_{\text {ind }}\right)^{n}\right\}
$$

where, $x_{\text {solid }}$ is the solid fraction of the sample at time $t$ during crystallisation (based on DSC peak area), $k$ is the rate constant, $t_{i n d}$ is the induction time and $n$ is the Avrami index related to the crystallisation mechanism. In logarithmic form the equation can be expressed as,

$$
\ln \left[-\ln \left(1-x_{\text {solid }}\right)\right]=\ln k+n \ln \left(t-t_{\text {ind }}\right)
$$

The slope and the intercept of the plot of $\ln \left[-\ln \left(1-x_{\text {solid }}\right)\right]$ versus $\ln \left(t-t_{\text {ind }}\right)$ provide the values of $n$ and $k$ respectively. These parameters can provide an insight into the crystallisation process. The constant $k$ can be thought of as a crystallisation rate constant and the Avrami index $n$ provides information of the type of nucleation (sporadic or pre-seeded) and the number of dimensions involved in crystal growth [29]. However, the units of $k$ are dependent on $n$. The time taken for half of the peak area of the main crystallisation peak to be reached $\left(t_{1 / 2}\right)$ after the apparent induction period can provide a better idea of the relative rates of crystallisation after nucleation, 


$$
t_{1 / 2}=\left(\frac{\ln 2}{k}\right)^{1 / n}
$$

ii) Stop-and-return technique: Samples were analysed further using the stop-and-return DSC technique [30] to provide a better picture of the evolution of the polymorphic state of the sample during isothermal crystallisation. The applied time-temperature program was as follows: holding at $80^{\circ} \mathrm{C}$ for 5 minutes to erase previous crystallisation memory, cooling at $25^{\circ} \mathrm{C} / \mathrm{min}$ to the isothermal crystallisation temperature $\left(20^{\circ} \mathrm{C}\right)$, holding for the required crystallisation time, and then heating at $10^{\circ} \mathrm{C} / \mathrm{min}$ to $80^{\circ} \mathrm{C}$. The crystallisation time before remelting was varied $(1,2,3,5,7,10,15,20$ and $30 \mathrm{~min})$. Two repetitions were performed for each time. The melting curves were integrated using a linear baseline.

iii) Non-isothermal crystallisation: Samples were heated initially at $80^{\circ} \mathrm{C}$ and held for 5 minutes in order to erase previous crystal memory. The samples were then cooled to $-20^{\circ} \mathrm{C}$ at $5^{\circ} \mathrm{C} / \mathrm{min}$ to crystallise the samples, and then heated to $60^{\circ} \mathrm{C}$ at $5^{\circ} \mathrm{C} / \mathrm{min}$ to view the subsequent melting profile.

\subsection{X-Ray diffraction}

In order to confirm the polymorphic identifications obtained via DSC, the crystalline phase transitions of stearin samples were also monitored by powder XRD using a Bruker D8 Advance diffractometer (Bruker AXS, UK) applying copper $\mathrm{K}$ alpha (CuKa) radiation with a wavelength of $1.5418 \AA$, and equipped with an Oxford Cryosystems Cryostream Plus low temperature apparatus (Oxford Cryosystems, Oxford, UK). Cooling of the samples was attained by a jet of liquid nitrogen. Temperature control was accurate to within $0.1^{\circ} \mathrm{C}$. Calibration of the diffraction angle was obtained by using silicon powder as a standard. Samples were melted at $80^{\circ} \mathrm{C}$ for 5 minutes to erase previous crystal memory, before cooling at $6^{\circ} \mathrm{C} / \mathrm{min}$ to $20^{\circ} \mathrm{C}$, and holding for 30 minutes. A slit focus reflection geometry was used and scans were measured every 1 minute over $2 \theta$ values between 15 and $30^{\circ}$. The XRD patterns were analysed for d-values using Diffrac. Eva software.

\subsection{Microstructure after storage}

Each sample ( 2 mg) was melted at $80^{\circ} \mathrm{C}$ and a drop was transferred onto a circular glass slide with the aid of a capillary tube. The drop was covered with a pre-heated cover slip before transferring to an incubator at $20^{\circ} \mathrm{C}$ and maintaining at this temperature for a period 
of 7 days. The crystalline microstructure of the samples was examined after 7 days using an Olympus BX43 polarised light microscope coupled to a Q-Imaging Retiga-2000R camera (Surrey, Canada) and Linksys 32 software data capture system (Linkam Instruments, Tadworth, UK).

\subsection{Raman Microscopy}

Polymorphic discrimination of the crystalline microstructures of stearin samples stored at $20^{\circ} \mathrm{C}$ for 7 days was performed using a Thermo Scientific DXR Raman Microscope (Thermo

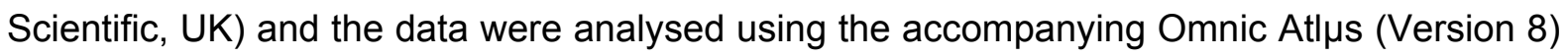
software following the method described by Ray et al. [21].

\subsection{Solid Fat Content (SFC)}

SFC was measured by pulsed NMR using a Bruker Minispec pc 20 (Bruker, Karlsruhe, Germany). Stearin samples were melted at $80^{\circ} \mathrm{C}$, placed in NMR tubes and submitted to the tempering treatments according to the IUPAC 2.150 serial tempered method as follows. The samples were maintained at $60^{\circ} \mathrm{C}$ for 20 min before cooling to $0^{\circ} \mathrm{C}$ and holding for $90 \mathrm{~min}$. Then the fat samples were stabilized at $26^{\circ} \mathrm{C}$ for $40 \mathrm{~h}$ and once again maintained at $0^{\circ} \mathrm{C}$ for $90 \mathrm{~min}$. The SFC was determined at $20^{\circ} \mathrm{C}, 30^{\circ} \mathrm{C}, 35^{\circ} \mathrm{C}$ and $40^{\circ} \mathrm{C}$ following 60 minute incubations at each temperature.

\section{Results and Discussion}

\subsection{Stearin compositions, yields and silica treatment}

Table 1 shows the TAG compositions of stearin fractions obtained after acetone fractionation. The samples are labelled according to the relative amounts of stearic and palmitic acid used in the original acidolysis reactions (ranging from 80:20 to 98:02), and also the purity of the stearic acid used (95\% or $98 \%$, labelled "S95" and "S98" respectively). The palmitic acid used was $98 \%$ pure in all cases.

All samples displayed a high Sat-O-Sat content (in the range $89.6 \%-93.1 \%$ ) with variations in StOSt (69.4 to $84 \%$ ) and POSt (4.5 to $20.7 \%$ ) content, whereas the POP content was found to be negligible $(<1 \%)$. This was due to the low amount of palmitic acid used in the acidolysis experiments. The amounts of StOSt increased and POSt decreased with 
increasing the ratio of St:P in the acidolysis reaction. The TAG compositions were very similar for samples of equivalent St:P ratios produced using the two stearic acid purities. A number of other minor TAG components, such as tristearoyl glycerol (StStSt) (1.1 to 3.2\%) and 1,3-distearoyl-2-linoleoyl-sn-glycerol (StLSt) (1.9 to $4.2 \%$ ) were also present in the stearin fractions. The presence of trisaturated TAGs i.e. StStSt can be attributed to acylmigration which might have occurred during the acidolysis reactions of HOSO. The yield of the stearin fractions varied from 30 to $35 \%$ of the weight of enzymatically modified fats (Table 1).

The compositions of the minor components, i.e. OX and DAGs present in the stearin fractions measured by HPLC are shown in Table 2. This shows it was possible to reduce the amounts of minor components significantly via silica treatment of the stearin fractions (Table 2). However, DAG and OX levels still remain significant (above $0.2 \%$ ) in some of the treated S98 samples. The untreated stearins were further analysed for individual DAG profile by HRGC using the silylation method. The results are shown in Table 3. The major DAG is 1,3StO, comprising over half of all DAGs, and is particularly prevalent in the $\$ 98$ samples. Also important are 1,2-StO (particularly in the S95 samples) and 1,2-PO and 1,3-PO in the samples with higher palmitic content. These DAG compositions mirror the overall prevalence of stearic, palmitic and oleic acids in the stearins.

\subsection{Isothermal crystallisation kinetics}

Fig. 1 shows isothermal $\left(20^{\circ} \mathrm{C}\right)$ crystallisation curves of the untreated and Si-treated stearins obtained by DSC. Each thermogram shows a major crystallisation exotherm, although in some cases an initial crystallisation also appeared to occur. The main evidence for this is the time taken for the DSC power to reach the new baseline during the transient period at the start of the isothermal period. Taking examples from Fig. 1A, the 95:05 and 90:10 samples (both Si-treated and untreated) take longer to reach the initial baseline than samples $85: 15$ and 80:20, and this may be due to crystallisation. Differences of this nature can also be seen between the untreated and treated samples for the first three S98 compositions in Fig 1B. Further evidence to support this comes from stop-and-return DSC data (see section 3.3), which show the a form on the remelt after holding for a period that is shorter than the induction time for the main peak. Also enthalpies reported in Table 4 for the main crystallisation peak are generally lower, for example samples with St:P ratios of 90:10 and higher. Some untreated $\mathbf{S 9 8}$ samples displayed a curious elbow shape on the initial transient suggesting a very slightly delayed initial crystallisation. 
To quantify the variation in the isothermal crystallisation kinetics of the samples, the solid fractions versus time data of the main exotherms were fitted to the Avrami equation by linear regression of $\ln \left[-\ln \left(1-x_{\text {solid }}\right)\right]$ vs $\ln \left(t-t_{\text {ind }}\right)$. The equation fitted the data very well over the whole range of solid fraction values with correlation coefficients higher than 0.98 . The kinetic parameters obtained are shown in Table 4.

The induction time $\left(t_{\text {ind }}\right)$ of the main peak, was generally slightly longer for the untreated stearin samples (for both S95 and S98) than that of the silica treated ones. The untreated samples also had a much greater variation of induction times, particularly for the S98 samples. This could reflect difficulties in producing stable nuclei due to the presence of the minor components, especially DAGs. There also appears to be a small effect of composition in that samples with higher palmitic acid content (higher POSt and lower StOSt) showed longer induction times.

The $t_{1 / 2}$ value provides a measure of the speed of the crystallisation and is related to the sharpness of the peaks. In general, stearin samples containing higher amounts of POSt exhibited longer induction times, lower $k$ values and higher $t_{1 / 2}$ values compared to the samples containing higher amounts of StOSt. Rousset et al. [31] have previously reported that POSt acts as a "brake" to the crystallisation of binary mixtures of POSt-StOSt, probably due to its asymmetric structure. Asymmetric molecules require more time to attach in the right position on a growing crystal and disrupt the packing and, consequently, can slow down the growth kinetics. The isothermal crystallisation kinetics of all the stearin samples (both untreated and Si-treated) reported in this work were faster when compared to commercial shea stearin [21]. However, the overall Sat-O-Sat content of the enzymatically produced stearins was much higher than that of shea stearin.

Silica treated samples showed greater variation however. In most cases treated samples showed shorter induction times but $t_{1 / 2}$ values showed a less distinct pattern. One pattern that seems to recur is that samples that displayed an initial crystallisation (on the starting transient) subsequently tended to have broader crystallisation peaks. The initial crystallisation therefore appears to "blunt" the later crystallisation. The variability observed with silica treated samples may be due to the relative amounts of minor components, which seem to still exert an influence even with a much reduced content. This will be further explored in the next section.

Untreated 98:02 (S98) and 95:05 (S98) stearins exhibited the shortest induction times and the sharpest peaks (Table 4$)$ in spite of containing high levels of DAGs $(5.3 \%$ and $4.8 \%$ respectively) and $\mathrm{OX}(0.7 \%$ and $1.6 \%$ respectively $)$. This is possibly a consequence of them 
containing a combination of high levels of 1,3-StO (promoting nucleation) and low levels of $1,2-\mathrm{StO}$.

\subsection{Stop-and-return DSC}

Fig. 2 illustrates the melting profiles of untreated and Si-treated S98 stearins obtained after holding at $20^{\circ} \mathrm{C}$ for various lengths of time using the "Stop-and-return" DSC method [30]. The equivalent S95 data can be viewed in Supporting Information (Fig. S1). The evolution of total melting enthalpy with time is also shown (for all samples) in Fig. 3. Three different melting peaks (at $22^{\circ} \mathrm{C}, 29^{\circ} \mathrm{C}$ and $33-36^{\circ} \mathrm{C}$ ) were generally observed for the untreated and

Si-treated stearin samples. Ray et al. [21] have previously reported very similar melting profiles for commercial shea stearin and several other studies $[32,33]$ have reported similar polymorphs for StOSt, namely $\alpha, \delta, y$ and $\beta$ ' with melting peak temperatures of $22.4,28.8$, $35.5^{\circ} \mathrm{C}$ and $36.5^{\circ} \mathrm{C}$ respectively. These correspond well to the peak temperatures found here for the stearins, although it is difficult to de-convolute melting of the $\gamma$ and $\beta^{\prime}$ forms as these overlap, so that either or both could comprise the highest melting peak. The final melting temperature of the stearins was found to very slightly decrease with increasing levels of POSt. A small melting peak at $\sim 41^{\circ} \mathrm{C}$ corresponding to the StOSt $\beta$ polymorph was also observed for the Si-treated 80:20 samples containing a high level of POSt. Bootello et al. [16] recently reported melting events for three different sunflower hard stearins evaluated via stop-and-return DSC. Although the TAG profiles of their stearins were different (lower StOSt and POSt levels and higher AOSt and BOSt) than that presented in this work, they also observed three peaks at similar temperatures to those seen here which they assigned to the melting of $\alpha, \beta$, and $\beta$ polymorphs based on the reasoning that these are commonly found in TAG systems. However, they did not report any XRD results for these samples.

It is apparent that some of the melting endotherms show a peak after only 1 minute of isothermal holding. The size of this peak correlates well with the incidence of the suspected initial crystallisation on the starting transient in the isothermal data in Fig. 1. This proves that there is an initial crystallisation of the $\alpha$ form, which is also accompanied by a small amount of $\delta$ formation. The incidence of $\alpha$ was greater at higher StOSt values, reducing as the POSt content increased until eventually disappearing at POSt levels above $15 \%$. This might be a direct effect of the POSt on the overall polymorphism of the stearins, as the melting temperature of the $\alpha$ polymorph of POSt has been reported to be below $20^{\circ} \mathrm{C}[32,34]$.

Some of the thermograms in Fig 2 then show an exothermic response (i.e. the signal is below the baseline) during the early part of the reheating process (leaving the initial transient 
aside). This must be due to either a continuation of crystallisation (likely in the case of the 0 minute samples) as the sample is heated or a recrystallisation of the $\alpha$ form into higher forms (likely with, for example, heating within 7 minutes of crystallisation). The $\alpha$ and $\delta$ forms persisted slightly longer (up to 5 minutes) in the untreated samples compared to the Sitreated samples (up to 3 minutes). This indicates an inhibiting effect of the DAGs on the rate of polymorphic transformation.

After 7 minutes almost all samples are in either the $y$ or $\beta^{\prime}$ forms. The overall progress of crystallisation can be seen in the enthalpy versus time plots in Fig. 3. These are consistent with the heights of the isothermal peaks shown in Fig. 1. The untreated 98:02 (S98) and 95:05 (S98) samples were found to have completely transformed into either the $Y$ and/or $\beta$ ' form within three minutes of crystallisation which was the fastest of all samples, and reflects the sharp isothermal DSC thermograms seen in Fig 1B. It may be that the lack of an initial $\alpha$ crystallisation leaves a greater supercooling and increased driving force for a later $\beta$ ' crystallisation.

There was some variation in the final values of the melting enthalpies which may be explained by different amounts of $\gamma$ and $\beta$ ' having formed. This can therefore be used to gauge the relative amounts of these two forms. It can be seen that higher final enthalpy values were obtained with the Si-treated samples (average 108.2 $\pm 1.4 \mathrm{~J} / \mathrm{g}$ and $113.2 \pm 4.3 \mathrm{~J} / \mathrm{g}$ for S95 and S98 respectively) compared to the untreated samples (with the exception of the 95:05 sample these averaged 100.1 $\pm 1.1 \mathrm{~J} / \mathrm{g}$ for $\mathrm{S95}$ and with the exception of the 98:02 sample averaged $105.6 \pm 4.1 \mathrm{~J} / \mathrm{g}$ for $\mathrm{S} 98$ samples). This, along with the slightly longer shoulder at low temperatures with the untreated samples suggests that the Si-treated samples may have had a greater conversion to $\beta^{\prime}$. A similar finding was made in the previous study on shea stearin [21].

\subsection{X-Ray diffraction}

The polymorphic identities of the stearins observed during the remelting of the stop-andreturn DSC were further validated by experiments carried out by XRD at $20^{\circ} \mathrm{C}$. It was quite likely that the polymorphism of the stearin samples will be similar to that of pure TAGs, such as StOSt and POSt, since these were found to be the main constituents of the fat samples (Table 1) [21]. Hence the diffraction patterns obtained from the samples were compared to literature XRD data for these pure TAGs [33, 34]. These studies found that $\alpha$ is characterised by a very strong peak at $4.21 \AA$, $\delta$ by a strong peak at $3.89 \AA$ and medium peaks at $4.11 \AA, 4.31 \AA, 4.63 \AA$ and $y$ by strong peaks at $3.88 \AA, 4.72 \AA$ and a medium peak 
at $4.50 \AA$ ( $\delta$ was only observed in impure samples of POSt and POP but not for StOSt). $\beta^{\prime}$ forms showed greater variations in the short spacings reported for the various TAGs with StOSt showing strong peaks at $3.70 \AA$ and $4.02 \AA$, whereas POSt showed strong peaks at $3.85 \AA$ and $4.13 \AA$. Both $\beta$ forms $\left(\beta_{1}\right.$ and $\left.\beta_{2}\right)$ were identifiable by a very strong peak for all TAGs at 4.58-4.61 $\AA$.

Fig. 4A-D shows the diffraction pattern of untreated and Si-treated 98:02 (S98) and 80:20 (S98) crystallised for different time intervals. The patterns for both untreated and Si-treated 98:02 (S98) (Fig. 4A and B) were very similar to each other. The first two scans were dominated by a peak at $4.16 \AA$. This did not correlate exactly with any literature diffractograms, but from its characteristic shape one can reasonably assign this to the $\alpha$ polymorph. We would expect the early patterns to include elements of the $\delta$ form also, which would present a peak at around $3.89 \AA$ for the $\delta$ component. A small peak can be seen at this position at 2 minutes and again at 3 minutes as a shoulder to another peak at $3.84 \AA$ which had suddenly appeared and is likely to correspond to the $y$ form. This corresponded with the stop-and-return DSC melting thermograms, where the $\delta$ melting peak was also prominently observed for the Si-treated 98:02 (S98) sample (Fig. 2). The $\delta$ form was not unequivocally observed for shea stearin by XRD by Ray et al. [21], and so this appears to be the first reported XRD evidence of the $\delta$ form in a high StOSt content system.

After 10 minutes, major diffraction peaks were identified for 98:02 samples at $4.65 \AA, 4.50 \AA$, $4.34 \AA, 4.17 \AA$ and $3.84 \AA$, which indicate the $y$ polymorph. Similar peaks (4.69 $\AA, 4.50 \AA$ and $3.89 \AA$ ) were also reported for shea stearin after 10 minutes of crystallisation [21]. After 30 minutes of crystallisation, strong diffraction peaks at $4.68 \AA, 4.51 \AA, 4.17 \AA$ and $3.85 \AA$ were observed, definitely indicating a mixture of the $Y$ and $\beta$ ' polymorphs (Fig. 4A and $B$ ). The particular peak at $4.67 \AA$ representing the $\mathrm{y}$ polymorph in the untreated sample was much more distinct compared to the Si-treated sample peak. This reflects the probability that the $y$ polymorph is present in higher amounts in the untreated samples in comparison to the Si-treated samples, which was suggested by the lower melting enthalpy data of the untreated samples observed in stop-and-return DSC experiments (Fig. 3C and D).

The untreated and Si-treated 80:20 (S98) stearins displayed different diffraction pattern profiles to each other and to the 98:02 stearins. The untreated 80:20 sample showed peaks at $4.16 \AA$ and $4.62 \AA$ up to 10 minutes of crystallisation which indicates patterns of a mixture of $\alpha$ and $\delta$ polymorphs (Fig. $4 \mathrm{C}$ ). This was similar to the melting events of the untreated 80:20 (S98) obtained by stop-and-return DSC (Fig. 2A). After 30 minutes of crystallisation diffraction peaks at $4.67 \AA, 4.50 \AA, 4.32 \AA, 4.20 \AA, 4.02 \AA, 3.87 \AA$ and $3.64 \AA$ signified the presence of a mixture of $y$ and $\beta$ ' polymorphs, but with a particularly high proportion of the $\gamma$ 
polymorph. In contrast, the Si-treated 80:20 (S98) stearin diffraction peaks (at $4.55 \AA$, 4.30 $\AA, 4.15 \AA$ and $3.87 \AA$ ) showed a combination of mainly $\delta$ and $y$ polymorphs, almost from the very first scan after 1 minute. If the stop-and-return DSC data of the Si-treated 80:20 (S98) stearin are carefully observed, the initial melting peaks (1 to 5 minutes) are also wide and correspond to a mixture of different polymorphs which supports the XRD patterns. After 30 minutes of crystallisation, diffraction peaks at $4.58 \AA, 4.35 \AA, 4.13 \AA, 3.88 \AA, 3.79 \AA$ and 3.67 $\AA$ indicated the presence of $\beta$ ' with traces of the $\beta$ polymorph which again corresponds with the stop-and-return DSC observation. In general, the XRD experiments are therefore broadly supportive of the Stop-and-return DSC results obtained for the stearin samples.

\subsection{Non-isothermal DSC thermograms}

Fig. 5 displays the DSC melting endotherms of the untreated and Si-treated S98 stearin fractions heated at $5^{\circ} \mathrm{C} / \mathrm{min}$ after previously cooling to $-20^{\circ} \mathrm{C}$ at $5^{\circ} \mathrm{C} / \mathrm{min}$ (the equivalent $\mathrm{S} 95$ data can be viewed in Fig. S2). Two principal melting peaks were observed for both sets of stearin fractions and a significant exothermic crystallisation event (between $\sim 25-30^{\circ} \mathrm{C}$ ) following (and overlapping) the first main peak. Judging by the stop-and-return thermograms and XRD analysis, the first peak can be attributed to the $\alpha$ form and the second peak to a combination of the $y$ and $\beta$ ' forms. A $\delta$ peak was not observed. The recrystallisation event was observed in all the samples, but was more prominent in the stearins with higher StOSt and lower POSt TAG content, and in those which were Si-treated. Similar polymorphic transitions of pure StOSt TAG were reported by Cebula and Smith [27] while heating at $5^{\circ} \mathrm{C} / \mathrm{min}$ after rapid cooling to $-10^{\circ} \mathrm{C}$. This transition occurs later (and at a higher temperature) for the untreated stearin fractions, which implies that the presence of DAGs and $O X$ in the samples had an inhibitory effect on recrystallisation. Ray et al. [21] also observed an inhibitory effect of DAGs on the recrystallisation of commercial shea stearin when subject to the same DSC programme.

\subsection{Microstructure and polymorphic form identification}

Fig. 6 displays the changes as observed by PLM that occurred in the microstructural network of the untreated and si-treated S98 stearin samples after crystallisation and subsequent storage for 7 days at $20^{\circ} \mathrm{C}$ (micrographs of S95 stearin samples can be viewed in Fig. S3). Morphological variations in crystal growth and polymorphic transformations of fat can be easily visualised using this technique. The same microscopic slides of the stearin samples were utilised to obtain the Raman spectra. Four different Raman spectra could be observed 
from the representative regions indicated by the different circles in Fig 6. Polymorphic identification was performed following the literature [35] and as described by Ray et al. [21]. Three different polymorphs were identified: (i) $\gamma$, corresponding to a dense structure of very small crystals (white dashed circle), (ii) $\beta$ ' which were pale spherulitic crystals (black dashed circle), and (iii) $\beta$ which were well defined spherulites (black solid circle). The fourth type of spectrum observed appeared to be a mixture of $\gamma$ and $\beta$ ', and was thus likely to be a mixture of the two forms (white solid circle). The a form was not observed.

The assignment of the Raman bands is shown in Table 5 and representative spectra are shown in Fig. 7 highlighting the main distinguishing features. The $\beta$ form was distinguished by a prominent peak at $1099 \mathrm{~cm}^{-1}$, whereas for other forms this was less prominent (attributed to disorder in the oleoyl chain segment). The $\beta$ form also exhibited two small peaks at $1254 \mathrm{~cm}^{-1}$ and $1275 \mathrm{~cm}^{-1}$ (indicated by arrows, Fig. 7B) rather than a single large peak which was displayed by $Y$ and $\beta^{\prime}$ spectra at $1261 \mathrm{~cm}^{-1}$ and $1263 \mathrm{~cm}^{-1}$ respectively. The $\beta^{\prime}$ form was distinguished by having a peak at $1423 \mathrm{~cm}^{-1}$ (Fig. 7B) arising from a splitting of the Raman active methylene scissoring mode and by a lack of a shoulder at $2900 \mathrm{~cm}^{-1}$ and $2860 \mathrm{~cm}^{-1}$ (Fig. 7A).

Differences in the three forms could also be seen in the $\mathrm{C}=\mathrm{O}$ stretching region (1720 to 1760 $\mathrm{cm}^{-1}$ ), which is shown expanded in Fig. $7 \mathrm{C}$ along with fits to a peak de-convolution algorithm. Whilst the $\beta^{\prime}$ form has a single major peak, the $\beta$ and $\gamma$ show two peaks but with different relative intensities. Whilst the higher wavenumber peak is stronger for $\beta$ it is the lower wavenumber peak which is stronger for $\gamma$. Similar observations were reported in the case of CB [36], StOSt (by conventional IR) [37] and tristearin [38]. However, Sprunt et al. [35] reported two bands for the $\beta$ form of StOSt.

With polymorphic assignments made the following observations can be made. Untreated samples showed both $Y$ and $\beta^{\prime}$ crystals (and the $Y+\beta^{\prime}$ ) mixture across almost all compositions. The $\beta$ polymorph was only observed in Si-treated samples. This again displays the inhibiting effect of the minor components in the crystal growth process. Untreated samples generally contained higher levels of DAGs (4.4\%, Table 2). The transformation of the untreated sample to higher polymorphic forms during stabilisation at $26^{\circ} \mathrm{C}$ might have been significantly hindered due to the presence of DAGs. Lower levels of DAGs and $O X$ in the Si-treated stearins prompted in the rapid transformation of the crystals to higher polymorphs. Shea stearin also exhibited similar large feathery spherulites at $20^{\circ} \mathrm{C}$ after 7 days [20]. The reported shea stearin spherulites were larger in size $(180-250 \mu \mathrm{m})$ in comparison to the spherulites observed in the Si-treated stearins. Shea stearin contains about $10 \%$ of liquid TAGs i.e. StLSt, StOO and $3 \%$ of long chain TAGs (AOSt), much higher 
in levels than that of the stearins reported in this work, which assists the rate and in the mobility during the crystallisation process to form large feathery spherulites.

Some minor variations with composition were also observed. As the level of palmitic acid (POSt) increased the $\beta$ spherulites appear larger and are also surrounded by a significant $\beta$ ' phase which was especially pronounced with the S95 samples (Fig. S3). This may also reflect a lower melting system with increased POSt levels.

\subsection{SFC Profiles}

Fig. 8 illustrates the SFC profiles of the untreated (A, C) and Si-treated $(B, D)$ stearin samples as measured by pulsed NMR. The SFC profiles of the stearin samples were highly influenced by respective TAG composition as well as the presence of minor components. With a few exceptions, samples with higher palmitic acid content showed a lower SFC profile, and this can be attributed to the relative amounts of POSt and StOSt (see Table 1, i.e. a higher POSt level depresses the SFC, particularly at $35^{\circ} \mathrm{C}$ ). However, samples that had been Si-treated showed a higher SFC profile (particularly at $35^{\circ} \mathrm{C}$ and $40{ }^{\circ} \mathrm{C}$ ) than untreated samples despite having similar TAG compositions. This likely to be due to a lack of complete transformation to the $\beta$ form for the untreated samples, due to the presence of DAGs or OX as seen in the microscope images (Fig. 6). There are correlations with the melting enthalpies shown in Fig 3 (stop-and-return DSC experiments) but these reflect the ability to form the $\beta^{\prime}$ polymorph whereas the SFC data reflect the ability to form $\beta$.

Samples made with the higher purity stearic acid also tended to produce slightly higher SFC values with all other factors remaining constant. The reason for this is not obvious as both had similar StOSt levels; however it may be linked to levels of minor TAGs such as higher levels of StLSt and AOSt in S95 samples, which might lead to a slight softening of the fat.

However, there are some discrepancies within the above trends, which may be explained by variations in the compositions of minor components. Firstly, for untreated samples the 98:02 (S98) and 95:05 (S98) stearins (Fig. 8C) exhibited lower SFC profiles compared to the 95:05 (S95) stearin (Fig. 8A) despite similar StOSt contents (Table 1). Secondly, for Si-treated S95 samples (Fig. 8B) the 90:10 sample has a higher SFC profile than 95:05, and with Si-treated S98 samples (Fig. 8D) the 95:05 sample has a higher SFC profile than the 98:02 sample. Again, both could be explained by lower levels of minor components in the samples. 


\section{Conclusions}

In this study nine hard stearin fractions were isolated via single stage acetone fractionation of acidolysed HOSO fats. The stearin samples contained varying amounts of StOSt (69$84 \%$ ) and POSt (4.5-21\%), with an overall Sat-O-Sat level of approximately $90 \%$. Significant amounts of oxidised glycerides and DAGs were present, particularly of 1,3-StO and so comparisons were performed on samples which were subject to an additional silica treatment to remove these minor components.

The effect of varying StOSt and POSt content was significant, in that crystallisation (at 20 ${ }^{\circ} \mathrm{C}$ ) speeded up as the StOSt level increased and the POSt level decreased, both in terms of the main crystallisation peak which produced forms $\gamma$ or $\beta^{\prime}$ but also in terms of an initial $\alpha$ crystallisation, which could occur at the very start of the isothermal period. Recrystallisation to higher forms was also faster with higher StOSt contents, and this was observed when heating after either isothermal $\left(20^{\circ} \mathrm{C}\right)$ and non-isothermal (constant cooling at $5^{\circ} \mathrm{C} / \mathrm{min}$ ) crystallisations. The composition effect is likely to be a result of difficulties of packing the asymmetric POSt molecule into predominantly StOSt crystals. The SFC profiles were also slightly reduced as POSt levels increased, due to the mixing effect of the lower melting POSt.

Polymorphic transformations from the lower forms such as $\alpha$ and $\delta$ to $\gamma$ and $\beta$ ' (particularly to $\left.\beta^{\prime}\right)$ were moderately promoted in both non-isothermal stop-and-return DSC experiments by the removal of minor components. However, subsequent transformation into the $\beta$ form was only observed after 7 days in the case of the Si-treated samples (confirmed via Raman microscopy), suggesting that the presence of the minor components in the untreated samples had a large inhibitory effect on the crystallisation to the higher polymorph. This was also found in an earlier study on shea stearin [21]. This explains differences between the SFC profiles of untreated and Si-treated samples, which showed that Si-treated stearins had higher solid contents (especially at $35^{\circ} \mathrm{C} \& 40^{\circ} \mathrm{C}$ ). There thus appears to be a more complete crystallisation of the highest polymorphic form $(\beta)$ during the stabilisation period for the Si-treated samples.

There appeared to be little difference in TAG compositions between stearins produced using $98 \%$ and $95 \%$ purity stearic acid in the original acidolysis reactions, but there were differences in the DAG compositions of untreated samples. $\$ 98$ samples showed a higher ratio of $1,3-\mathrm{StO}$ to $1,2-\mathrm{StO}$, and this appeared to promote faster crystallisation of the $y$ and $\beta$ ' forms after initially suppressing formation of the $\alpha$ form. 


\section{Acknowledgements}

This research was sponsored by IOI Loders Croklaan Europe and Loughborough University, UK. Thanks are extended to Mr. Nick J. Spencer at the University of Reading for the XRD analyses and to Mr. Jun Ma at Loders Croklaan for the Solid Fat Content and DAG content analyses. The authors would also like to acknowledge the European Research Council (Grant number 280106-CrySys) for funding the Thermo Scientific DXR Raman Microscope.

\section{References}

[1] Gunstone, F. D. (1997) Major Sources of Lipids. In: Gunstone F. D., Padley F. B. (eds) Lipid Technologies and Applications. Marcel Dekker Inc., New York, pp 1-18.

[2] Padley, F. B. (1997) Chocolate and Confectionary Fats. In: Gunstone, F. D., Padley, F. B. (eds) Lipid Technologies and Applications. Marcel Dekker Inc., New York, pp 391432.

[3] Chaiseri, S., Dimick, P. S. (1989) Lipid and Hardness Characteristics of Cocoa Butters from Different Geographic Regions. J. Am. Oil Chem. Soc. 66, 1771-1776.

[4] Foubert, I., Vanrolleghem, P., Thas, O., Dewettinck, K., Influence of chemical composition on the isothermal cocoa butter crystallization. J. Food Sci. 2004, 69, 478487.

[5] Smith, K. W., (2001) Cocoa Butter and Cocoa Butter Equivalents. In: Gunstone FD (ed) Structured and Modified Lipids. Marcel Dekker, Inc., New York, pp 401-422.

[6] Lipp, M., Anklam, E., Review of Cocoa Butter and Alternative Fats for Use in Chocolate - Part A. Compositional data. Food Chem. 1998, 62, 73-97.

[7] Alander, J., Shea butter - A multifunctional ingredient for food and cosmetics. Lipid Technol. 2004, 16, 202-205.

[8] T. Kawada: US patent 4268538 (1981).

[9] I. Kazuo: European patent $0307493 A 1$ (1989).

[10] H. Maeda: US patent 4882192 (1989).

[11] T. Okada: European patent 1038444A1 (2000).

[12] J. H. Kang: International publication number WO 2010/053244A1 (2010)

[13] T. L. H. Favre: International publication number WO 2010/130395A1 (2010)

[14] Salas, J. J., Bootello, A. M., Martínez-Force, E., Garcés. R., Production of stearate-rich butters by solvent fractionation of high stearic-high oleic sunflower oil. Food Chem. 2011, 124, 450-458. 
[15] Bootello, A. M., Hartel, R. W., Garcés. R., Martínez-Force, E., Salas, J. J., Evaluation of high oleic-high stearic sunflower hard stearins for cocoa butter equivalent formulation. Food Chem. 2012, 134, 1409-1417.

[16] Bootello, A. M., Hartel, R. W., Levin, M., Martínez-Blanes, J. M., Real, C., Garcés. R., Martínez-Force, E., Salas, J. J., Studies of isothermal crystallisation kinetics of sunflower hard stearin-based confectionery fats. Food Chem. 2013, 139, 184-195.

[17] Ray, J., Nagy, Z. K., Smith, K. W., Bhaggan, K., Stapley, A. G. F., Kinetic study of the acidolysis of High Oleic Sunflower Oil with Stearic-Palmitic acid mixtures catalysed by immobilised Rhizopus oryzae lipase, Biochem. Eng. J. 2012, 73, 17-28.

[18] Smith, K. W., Bhaggan, K., Talbot, G., van Malssen, K. F., Crystallization of fats: Influence of minor components and additives. J. Am. Oil Chem. Soc. 2011, 88, 10851101.

[19] Siew, W. L., Ng, W. L., Effect of diglycerides on the crystallisation of palm oleins. J. Sci. Food Agric. 1996, 726, 496-500.

[20] Siew, W. L., Ng, W. L., Influence of diglycerides on crystallisation of palm oil. J. Sci. Food Agric. 1999, 726, 722-726.

[21] Ray, J., Nagy, Z. K., Smith, K. W., Bhaggan, K., Stapley, A. G. F., Crystallization and polymorphic behaviour of shea stearin and the effect of removal of polar components. Eur. J. Lipid Sci. Technol. 2013, 115, 1094-1106.

[22] Wright, A. J., Hartel, R. W., Narine, S. S., Marangoni, A. G., The effect of minor components on milk fat crystallization. J. Am. Oil Chem. Soc. 2000, 77, 463-475.

[23] Wright, A. J., Marangoni, A. G., Effect of DAG on milk fat TAG crystallization. J. Am. Oil Chem. Soc. 2002, 79, 395-402.

[24] Cheong, L. Z., Zhang, H., Xu, Y., Xu, X., Physical characterization of lard partial acylglycerols and their effects on melting and crystallization properties of blends with rapeseed oil. J. Agric. Food Chem. 2009, 57, 5020-5027.

[25] Saitou, K., Mitsui, Y., Shimizu, M., Kudo, N., Katsuragi, Y., Sato, K., Crystallization behaviour of diacylglycerol-rich oils produced from rapeseed oil. J. Am. Oil Chem. Soc. 2012, 89, 1231-1239.

[26] Wähnelt, S., Teusel, D., Tülsner, M., Influence of isomeric diglycerides on phase transition of cocoa butter-investigation by isothermal DSC. Fat Sci. Technol. 1991, 93, 174-178.

[27] Cebula, D. J., Smith, K. W., Differential Scanning Calorimetry of Confectionery Fats: Part II - Effects of Blends and Minor Components. J. Am. Chem. Soc. 1992, 69, 992998.

[28] Avrami, M., Kinetics of phase change. II. Transformation-time relations for random distribution of nuclei. J. Am. Oil Chem. Soc. 1940, 8, 212-224. 
[29] Marangoni, A. G., Crystallization kinetics. In: Fat crystal networks. Eds. Marangoni, A. G., Marcel Dekker Inc, New York (USA) 2005, pp. 21-82.

[30] Foubert, I., Fredrick, E., Vereecken, J., Sichien, M., Dewettinck, K., Stop-and-return DSC method to study fat crystallization. Thermochim. Acta. 2008, 471, 7-13.

[31] Rousset, Ph., Rappaz, M., Minner, E., Polymorphism and solidification kinetics of the binary system POS-SOS. J. Am. Oil Chem. Soc. 1998, 75, 857-864.

[32] Rousset, P., Rappaz, M., Crystallization Kinetics of the Pure Triacylglycerols Glycerol1,3-Dipalmitate-2-Oleate, Glycerol- 1-Palmitate-2-Oleate-3-Stearate, and Glycerol-1,3 Distearate- 2-Oleate. J. Am. Oil Chem. Soc.1996, 73, 1051-1057.

[33] Sato, K., Arishima, T., Wang, Z. H., Ojima, K., Sagi, N., Mori, H., Polymorphism of POP and SOS. I. Occurrence and Polymorphic Transformation, J. Am. Oil Chem. Soc. 1989, 66, 664-674.

[34] Arishima, T., Sagi, N., Mori, H., Sato, K., Polymorphism of POS. I. Occurrence and polymorphic transformation, J. Am. Oil Chem. Soc. 1991, 68, 710-715.

[35] Sprunt, J. C., Jayasooriya, U. A., Wilson, R. H., A simultaneous FT-Raman-DSC (SRD) study of polymorphism in sn-1,3-distearoyl-2-oleoylglycerol (SOS). Phys. Chem. Chem. Phys. 2000, 2, 4299-4305.

[36] Bresson, S., Rousseau, D., Ghosh, S., Marssi, E. M., Faivre, V., Raman Spectroscopy of the polymorphic forms and liquid state of cocoa butter. Eur. J. Lipid Sci. Technol. 2011, 113, 992-1004.

[37] Yano, J., Ueno, S., Sato, K., Arishima, T., Sagi, N., Kaneko, F., Kobayashi, M., FT-IR Study of Polymorphic Transformations in SOS, POP, and POS. J. Phys. Chem. 1993, 97, 12967-12973.

[38] Silva, E. D., Bresson, S., Rousseau, D., Characterization of the three major polymorphic forms and liquid state of tristearin by Raman spectroscopy. Chem. Phys. Lipids 2009, 157, 113-119. 


\section{List of Figures}

Figure 1. Comparison of DSC isothermal crystallisation profiles of untreated and Si-treated (A) S95 and (B) S98 stearins at a crystallisation temperature of $20^{\circ} \mathrm{C}$. Crystallisation exotherms were obtained by heating the samples at $80^{\circ} \mathrm{C}$ for 5 minutes, cooling to $20^{\circ} \mathrm{C}$ at $25^{\circ} \mathrm{C} / \mathrm{min}$ and holding for 30 minutes. Approximate induction times of the main crystallisation peak $\left(t_{\text {ind }}\right)$ are indicated by arrows on the exotherms.

Figure 2. Melting profiles indicating polymorphic forms $\left(\alpha, \delta, \gamma\right.$ and $\left.\gamma^{+} \beta^{\prime}\right)$ of $(A)$ untreated and (B) Si-treated $S 98$ stearins obtained by the stop-and-return DSC method in which the isothermal crystallisation at $20^{\circ} \mathrm{C}$ was interrupted after $1,2,3,5,7,10,15,20$ and 30 minutes.

Figure 3. Comparison of the melting enthalpies as a function of time for the isothermal crystallisation at $20^{\circ} \mathrm{C}$ of (A) untreated S95, (B) Si-treated S95, (C) untreated S98 and (D) Si-treated $S 98$ stearins obtained by the stop-and-return DSC method.

Figure 4. XRD patterns of (A) untreated 98:02 (S98), (B) Si-treated 98:02 (S98), (C) untreated 80:20 (S98) and (D) Si-treated 80:20 (S98) stearins crystallised at $20^{\circ} \mathrm{C}$ for different times.

Figure 5. Non-isothermal DSC heating thermograms of untreated and Si-treated S98 stearins. Melting exotherms were obtained by heating the samples to $60^{\circ} \mathrm{C}$ at $5^{\circ} \mathrm{C} / \mathrm{min}$ minutes after previously cooling from $80^{\circ} \mathrm{C}$ to $-20^{\circ} \mathrm{C}$ at $5^{\circ} \mathrm{C} / \mathrm{min}$.

Figure 6. Microstructure of (A) untreated and (B) Si-treated S98 stearins acquired under polarised light after crystallising at $20^{\circ} \mathrm{C}$ for 7 days. Different polymorphic forms (identified by Raman microscopy) are indicated by circles outlined on the microstructure. The white dashed circle represents the $\gamma$ form, white solid circle represents the $\gamma^{+} \beta$ ' forms, black dashed circle represents the $\beta$ ' form and black solid circle represents the $\beta$ form.

Figure 7. Representative Raman spectra for stearins in the following ranges: (A) 3000-2700 $\mathrm{cm}^{-1}$, (B) $1800-800 \mathrm{~cm}^{-1}$, and (C) $1780-1700 \mathrm{~cm}^{-1}$ (magnified $\mathrm{C}=\mathrm{O}$ stretching region). The four spectra types correspond to the microstructure indicated by circles in Fig. 6 and are identified as: (a) $y$ form, (b) mixture of $y$ and $\beta^{\prime}$ forms (c) $\beta^{\prime}$ form and (d) $\beta$ form. Significant differences between the polymorphs are indicated by arrows on the Raman spectra.

Figure 8. SFC profiles of the (A) untreated S95, (B) Si-treated S95, (C) untreated S98, and (D) Si-treated S98 stearins. 
Table 1. Triacylglycerol composition (wt\%) and yield (\%) of the fractionated and Si-treated stearin samples as measured by HRGC.

\begin{tabular}{|c|c|c|c|c|c|c|c|c|c|}
\hline \multirow{2}{*}{ TAGs } & \multicolumn{9}{|c|}{ Stearin samples } \\
\hline & 95:05 (S95) & $90: 10$ (S95) & 85:15 (S95) & $80: 20$ (S95) & 98:02 (S98) & 95:05 (S98) & 90:10 (S98) & $85: 15$ (S98) & $80: 20$ (S98) \\
\hline PPSt & 0 & 0.1 & 0.2 & 0.3 & 0 & 0.1 & 0.1 & 0.2 & 0.4 \\
\hline POP & 0.1 & 0.1 & 0.4 & 0.9 & 0.1 & 0.1 & 0.2 & 0.3 & 0.8 \\
\hline PLP & 0 & 0 & 0 & 0.2 & 0.1 & 0 & 0 & 0 & 0 \\
\hline PStSt & 0.5 & 1 & 0.8 & 1.3 & 0.4 & 0.7 & 1.2 & 1.6 & 2.1 \\
\hline POSt & 5.2 & 9.8 & 15.7 & 20.7 & 4.5 & 6.1 & 11.2 & 14.2 & 17.9 \\
\hline POO & 0.1 & 0.1 & 0.1 & 0.1 & 0.1 & 0.1 & 0.1 & 0.3 & 0.2 \\
\hline PLSt & 0.1 & 0.2 & 0.2 & 0.3 & 0.1 & 0.1 & 0.2 & 0.1 & 0.1 \\
\hline PLO & 0.1 & 0 & 0.2 & 0.1 & 0 & 0 & 0 & 0 & 0 \\
\hline StStSt & 3.2 & 2.9 & 1.1 & 1.3 & 3.3 & 3.2 & 2.9 & 2.3 & 2.7 \\
\hline StOSt & 83.8 & 79 & 75.6 & 69.4 & 83.7 & 84 & 78.4 & 76 & 71.3 \\
\hline StOO & 1.1 & 1.3 & 1 & 0.8 & 1.9 & 1.2 & 0.9 & 1.3 & 0.8 \\
\hline StLSt & 4.1 & 3.9 & 3.3 & 2.8 & 4.2 & 2.9 & 3.3 & 1.9 & 1.9 \\
\hline 000 & 0 & 0 & 0 & 0 & 0 & 0.1 & 0 & 0.2 & 0.1 \\
\hline OLO & 0 & 0 & 0 & 0.1 & 0.1 & 0.1 & 0.1 & 0 & 0 \\
\hline StLL & 0 & 0 & 0 & 0 & 0 & 0.1 & 0 & 0 & 0 \\
\hline AStSt & 0.1 & 0.1 & 0 & 0.1 & 0.1 & 0.1 & 0.1 & 0.1 & 0.1 \\
\hline AOSt & 1.5 & 1.5 & 1.5 & 1.5 & 1.3 & 1.2 & 1.2 & 1.3 & 1.4 \\
\hline Sum Sat-O-Sat & 90.6 & 90.4 & 93.1 & 92.5 & 89.6 & 91.4 & 91.0 & 91.8 & 91.4 \\
\hline Stearin Yield (\%) & 33.5 & 32 & 30.5 & 30.2 & 35 & 31.5 & 33.5 & 31 & 32.2 \\
\hline
\end{tabular}


Table 2. Composition of minor components (wt\%) in the stearin samples as measured by HPLC, where A represents untreated stearins and B represents Si-treated stearins.

\begin{tabular}{|c|c|c|c|c|c|c|c|c|c|c|c|c|c|c|c|c|c|c|}
\hline \multirow{3}{*}{$\begin{array}{l}\text { Minor } \\
\text { Components }\end{array}$} & \multicolumn{18}{|c|}{ Stearin samples } \\
\hline & \multicolumn{2}{|c|}{ 95:05 (S95) } & \multicolumn{2}{|c|}{$90: 10$ (S95) } & \multicolumn{2}{|c|}{$85: 15$ (S95) } & \multicolumn{2}{|c|}{$80: 20$ (S95) } & \multicolumn{2}{|c|}{ 98:02 (S98) } & \multicolumn{2}{|c|}{ 95:05 (S98) } & \multicolumn{2}{|c|}{$90: 10$ (S98) } & \multicolumn{2}{|c|}{$85: 15$ (S98) } & \multicolumn{2}{|c|}{$80: 20$ (S98) } \\
\hline & $A$ & $\mathrm{~B}$ & $A$ & $B$ & $A$ & $\mathrm{~B}$ & A & $\mathrm{B}$ & $A$ & $B$ & $A$ & $B$ & $A$ & $\mathrm{~B}$ & $A$ & $\mathrm{~B}$ & $A$ & $\mathrm{~B}$ \\
\hline OX & 0.1 & 0 & 0.2 & 0 & 0.9 & 0 & 1.4 & 0 & 0.7 & 0.1 & 1.6 & 0.1 & 0.7 & 0.1 & 0.8 & 0.1 & 2 & 0 \\
\hline 1,2-DAG & 0.8 & 0 & 0.4 & 0 & 0.4 & 0 & 0.5 & 0 & 0.4 & 0 & 0.4 & 0 & 0.2 & 0 & 0.2 & 0 & 0.4 & 0 \\
\hline 1,3-DAG & 3.7 & 0.2 & 3.5 & 0.1 & 3.8 & 0.1 & 3.9 & 0.1 & 4.9 & 0.9 & 4.4 & 0.4 & 4.4 & 0.5 & 4.9 & 0.4 & 5.2 & 0 \\
\hline Total DAG & 4.5 & 0.2 & 3.9 & 0.1 & 4.2 & 0.1 & 4.4 & 0.1 & 5.3 & 0.9 & 4.8 & 0.4 & 4.6 & 0.5 & 5.1 & 0.4 & 5.6 & 0 \\
\hline
\end{tabular}


Table 3. Individual DAG compositions as a percentage of total DAGs in the untreated stearins, as measured by HR-GC.

\begin{tabular}{|c|c|c|c|c|c|c|c|c|c|}
\hline \multirow{2}{*}{ DAGs } & \multicolumn{9}{|c|}{ Stearin samples } \\
\hline & 95:05 (S95) & 90:10 (S95) & $85: 15$ (S95) & $80: 20$ (S95) & 98:02 (S98) & 95:05 (S98) & 90:10 (S98) & $85: 15$ (S98) & $80: 20$ (S98) \\
\hline $1,2-\mathrm{PO}$ & 2.1 & 3.0 & 3.8 & 3.0 & 0.0 & 0.0 & 1.2 & 1.3 & 1.6 \\
\hline 1,3-PO & 3.6 & 7.6 & 12.6 & 15.5 & 3.6 & 4.9 & 9.4 & 17.2 & 17.0 \\
\hline 1,3-PL & 0.0 & 0.0 & 0.0 & 0.0 & 0.0 & 0.0 & 0.0 & 0.0 & 0.0 \\
\hline 1,3-PLn & 0.0 & 2.1 & 2.0 & 2.5 & 1.5 & 2.1 & 1.6 & 1.8 & 1.0 \\
\hline $1,2-S t S t$ & 0.0 & 2.9 & 3.2 & 2.7 & 1.5 & 2.3 & 2.1 & 1.7 & 1.6 \\
\hline $1,2-\mathrm{StO}$ & 30.8 & 26.7 & 18.7 & 16.2 & 15.6 & 12.7 & 11.8 & 7.1 & 8.7 \\
\hline $1,2-00$ & 1.1 & 2.0 & 2.6 & 1.5 & 1.9 & 1.3 & 1.2 & 1.0 & 0.9 \\
\hline $1,3-\mathrm{StO}$ & 58.8 & 49.8 & 50.6 & 56.1 & 69.8 & 72.8 & 70.0 & 66.8 & 66.9 \\
\hline $1,2-\mathrm{OL}$ & 3.5 & 5.9 & 6.3 & 2.5 & 6.0 & 3.7 & 2.7 & 3.2 & 2.3 \\
\hline
\end{tabular}


Table 4. Induction time $\left(t_{\text {ind }}\right)$, Avrami index $(n)$, isothermal rate constant $(k)$, half-time of crystallisation $\left(t_{1 / 2}\right)$ and isothermal crystallisation enthalpy $[\mathrm{J} / \mathrm{g}]$ for untreated stearins and Sitreated stearins at $20^{\circ} \mathrm{C}$

\begin{tabular}{lllllll}
\hline Stearin samples & $t_{\text {ind }}{ }^{a}(\mathrm{~min})$ & $n^{a}$ & $k^{a}\left(\mathrm{~min}^{-1}\right)$ & $t_{1 / 2}{ }^{a}(\mathrm{~min})$ & $R^{2}$ & $\begin{array}{l}\text { Crystallisation } \\
\text { Enthalpy }^{a}(\mathrm{~J} / \mathrm{g})\end{array}$ \\
\hline Untreated & & & & & & \\
\hline $95: 05$ (S95) & $3.73 \pm 0.02$ & $2.62 \pm 0.02$ & $9.38 \times 10^{-2} \pm 0.40 \times 10^{-2}$ & $2.14 \pm 0.04$ & 0.99 & $36.36 \pm 0.43$ \\
$90: 10$ (S95) & $4.25 \pm 0.06$ & $3.16 \pm 0.08$ & $3.98 \times 10^{-2} \pm 1.02 \times 10^{-2}$ & $2.49 \pm 0.16$ & 0.98 & $59.76 \pm 0.62$ \\
$85: 15$ (S95) & $4.80 \pm 0.12$ & $3.23 \pm 0.10$ & $1.89 \times 10^{-2} \pm 0.45 \times 10^{-2}$ & $3.08 \pm 0.21$ & 0.98 & $70.65 \pm 1.60$ \\
$80: 20$ (S95) & $5.21 \pm 0.09$ & $3.01 \pm 0.08$ & $2.06 \times 10^{-2} \pm 0.67 \times 10^{-2}$ & $3.27 \pm 0.30$ & 0.99 & $63.79 \pm 1.06$ \\
$98: 02$ (S98) & $2.66 \pm 0.05$ & $3.34 \pm 0.13$ & $51.49 \times 10^{-2} \pm 11.0 \times 10^{-2}$ & $1.10 \pm 0.07$ & 0.98 & $53.19 \pm 0.10$ \\
$95: 05$ (S98) & $2.86 \pm 0.05$ & $3.34 \pm 0.14$ & $21.36 \times 10^{-2} \pm 3.32 \times 10^{-2}$ & $1.43 \pm 0.04$ & 0.99 & $54.93 \pm 0.16$ \\
$90: 10$ (S98) & $4.57 \pm 0.02$ & $3.06 \pm 0.18$ & $5.78 \times 10^{-2} \pm 0.24 \times 10^{-2}$ & $2.26 \pm 0.12$ & 0.98 & $65.70 \pm 1.20$ \\
$85: 15$ (S98) & $4.27 \pm 0.03$ & $2.74 \pm 0.05$ & $1.47 \times 10^{-2} \pm 0.10 \times 10^{-2}$ & $4.07 \pm 0.05$ & 0.99 & $69.45 \pm 0.33$ \\
$80: 20$ (S98) & $8.55 \pm 0.12$ & $3.07 \pm 0.14$ & $0.38 \times 10^{-2} \pm 0.15 \times 10^{-2}$ & $5.17 \pm 0.14$ & 0.98 & $66.26 \pm 0.81$ \\
\hline Si-treated & & & & & & \\
\hline $95: 05$ (S95) & $3.45 \pm 0.03$ & $2.77 \pm 0.11$ & $9.89 \times 10^{-2} \pm 0.30 \times 10^{-2}$ & $2.05 \pm 0.05$ & 0.99 & $36.00 \pm 0.23$ \\
$90: 10$ (S95) & $3.91 \pm 0.03$ & $2.72 \pm 0.05$ & $4.68 \times 10^{-2} \pm 0.20 \times 10^{-2}$ & $2.69 \pm 0.05$ & 0.99 & $49.50 \pm 0.68$ \\
$85: 15$ (S95) & $4.49 \pm 0.04$ & $2.96 \pm 0.09$ & $2.94 \times 10^{-2} \pm 0.26 \times 10^{-2}$ & $2.91 \pm 0.10$ & 0.98 & $75.42 \pm 0.51$ \\
$80: 20$ (S95) & $3.95 \pm 0.03$ & $2.61 \pm 0.06$ & $2.99 \times 10^{-2} \pm 0.30 \times 10^{-2}$ & $3.33 \pm 0.05$ & 0.99 & $58.61 \pm 1.27$ \\
$98: 02$ (S98) & $3.37 \pm 0.03$ & $2.65 \pm 0.05$ & $13.55 \times 10^{-2} \pm 0.81 \times 10^{-2}$ & $1.85 \pm 0.03$ & 0.99 & $41.02 \pm 0.44$ \\
$95: 05$ (S98) & $3.47 \pm 0.05$ & $3.01 \pm 0.16$ & $18.82 \times 10^{-2} \pm 4.84 \times 10^{-2}$ & $1.56 \pm 0.14$ & 0.98 & $59.45 \pm 0.39$ \\
$90: 10$ (S98) & $3.96 \pm 0.03$ & $2.46 \pm 0.08$ & $3.61 \times 10^{-2} \pm 0.75 \times 10^{-2}$ & $3.35 \pm 0.17$ & 0.99 & $43.65 \pm 0.66$ \\
$85: 15$ (S98) & $3.77 \pm 0.02$ & $3.07 \pm 0.08$ & $4.27 \times 10^{-2} \pm 0.31 \times 10^{-2}$ & $2.48 \pm 0.07$ & 0.98 & $73.66 \pm 0.60$ \\
$80: 20$ (S98) & $5.40 \pm 0.14$ & $3.04 \pm 0.07$ & $0.92 \times 10^{-2} \pm 0.22 \times 10^{-2}$ & $4.16 \pm 0.20$ & 0.98 & $63.41 \pm 0.74$ \\
\hline
\end{tabular}

${ }^{a}$ Values represent the mean \pm standard deviation of three replicates 
Table 5. Raman scattered bands for the polymorphs observed for untreated stearins and Sitreated stearins stored at $20^{\circ} \mathrm{C}$ for 7 days

\begin{tabular}{|c|c|c|c|c|}
\hline Vibration & $y$ & $\gamma+\beta^{\prime}$ & $\beta^{\prime}$ & $\beta$ \\
\hline $\mathrm{r}\left(\mathrm{CH}_{3}\right)$ & $892(\mathrm{~m})$ & 892(m) & $891(\mathrm{~m})$ & $891(\mathrm{~m})$ \\
\hline $\mathrm{r}\left(\mathrm{CH}_{3}\right)$ & $924(w)$ & $924(w)$ & $924(w)$ & $924(w)$ \\
\hline $\mathrm{r}\left(\mathrm{CH}_{3}\right)$ & - & - & - & $940(w)$ \\
\hline $\mathrm{r}\left(\mathrm{CH}_{3}\right)$ & $972(w)$ & $971(w)$ & $968(m)$ & $960(v w)$ \\
\hline $\mathrm{V}_{\mathrm{as}}(\mathrm{C}-\mathrm{C}) \mathrm{T}$ & 1062(s) & 1064(s) & $1064(\mathrm{~s})$ & $1063(\mathrm{~s})$ \\
\hline$v_{s}(C-C) G$ & $1100(w)$ & 1102(w) & $1101(w)$ & 1099(w) \\
\hline $\mathrm{v}_{\mathrm{s}}(\mathrm{C}-\mathrm{C}) \mathrm{T}$ & $1131(\mathrm{~s})$ & $1131(\mathrm{~s})$ & $1130(\mathrm{~s})$ & 1131(s) \\
\hline $\mathrm{r}\left(\mathrm{CH}_{2}\right)$ & $1177(\mathrm{~m})$ & $1176(\mathrm{vw})$ & 1175(vw) & 1177(vw) \\
\hline $\mathrm{T}\left(\mathrm{CH}_{2}\right)$ & $1261(\mathrm{~m})$ & $1264(\mathrm{~m})$ & 1263(m) & $1254(w)$ \\
\hline $\mathrm{T}\left(\mathrm{CH}_{2}\right)$ & - & 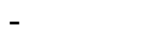 & - & $1275(\mathrm{~s})$ \\
\hline $\mathrm{T}\left(\mathrm{CH}_{2}\right)$ & $1297(s)$ & 1297(s) & 1297(s) & $1297(\mathrm{~s})$ \\
\hline$\delta(C-H)$ & $1371(w)$ & 1372(vw) & 1374(vw) & $1370(\mathrm{vw})$ \\
\hline$\delta\left(\mathrm{CH}_{2}\right)$ & - & $1423(\mathrm{vw})$ & 1423(m) & - \\
\hline$\delta\left(\mathrm{CH}_{2}\right)$ & 1443(s) & 1441(s) & $1440(s)$ & 1443(s) \\
\hline$\delta_{a}\left(\mathrm{CH}_{3}\right)$ & $1466(m)$ & $1457(w)$ & 1459(m) & $1465(\mathrm{~m})$ \\
\hline$v_{s}(C=C)$ & $1655(\mathrm{~m})$ & 1656(m) & 1655(m) & $1661(\mathrm{~m})$ \\
\hline$v(C=O)$ & $1736(\mathrm{~m})$ & 1736(m) & 1737(m) & 1736(m) \\
\hline$v(C=O)$ & $1750(w)$ & 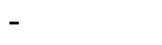 & 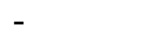 & $1745(w)$ \\
\hline $\mathrm{v}\left(\mathrm{CH}_{3}-\mathrm{CH}_{2}\right)$ & $2727(\mathrm{~m})$ & $2725(\mathrm{~m})$ & $2727(\mathrm{~m})$ & $2728(m)$ \\
\hline $\mathrm{V}_{\mathrm{s}}\left(\mathrm{CH}_{2}\right)$ & 2850 (s) & $2849(\mathrm{~s})$ & $2849(\mathrm{~s})$ & $2848(s)$ \\
\hline $\mathrm{V}_{\mathrm{s}}\left(\mathrm{CH}_{2}\right)$ & $2860(w)$ & - & - & $2860(w)$ \\
\hline $\mathrm{V}_{\text {as }}\left(\mathrm{CH}_{2}\right)$ & 2882(s) & 2882(s) & 2882(s) & 2882(s) \\
\hline $\mathrm{V}_{\mathrm{as}}\left(\mathrm{CH}_{2}\right)$ & 2906(sh) & 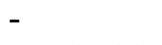 & - & 2902(sh) \\
\hline $\mathrm{v}_{\mathrm{s}}\left(\mathrm{CH}_{3}\right)$ & 2932(sh) & 2931(sh) & 2925(sh) & 2931(sh) \\
\hline $\mathrm{V}_{\mathrm{as}}\left(\mathrm{CH}_{3}\right)$ & 2965(sh) & 2962(sh) & 2963(sh) & 2958(sh) \\
\hline $\mathrm{v}(\mathrm{C}-\mathrm{H})$ & $3006(w)$ & $3005(w)$ & $3005(w)$ & $3005(w)$ \\
\hline
\end{tabular}

vw, very weak; $w$, weak; $m$, medium; s, strong;

sh, shoulder; v, stretch; $\delta$, deformation; $\mathrm{T}$, twist; r, rock; s, symmetric; as, antisymmetric; T, trans; G, gauche. Units are $\mathrm{cm}^{-1}$. 

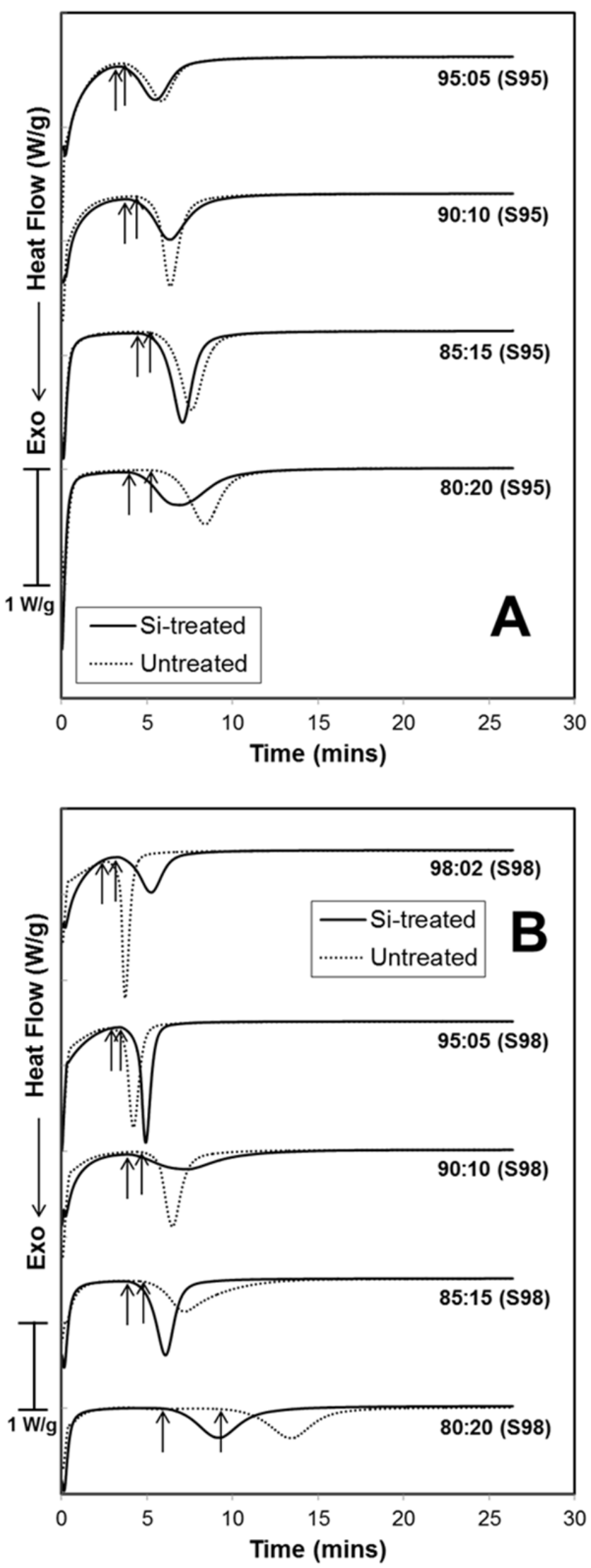

Figure 1 

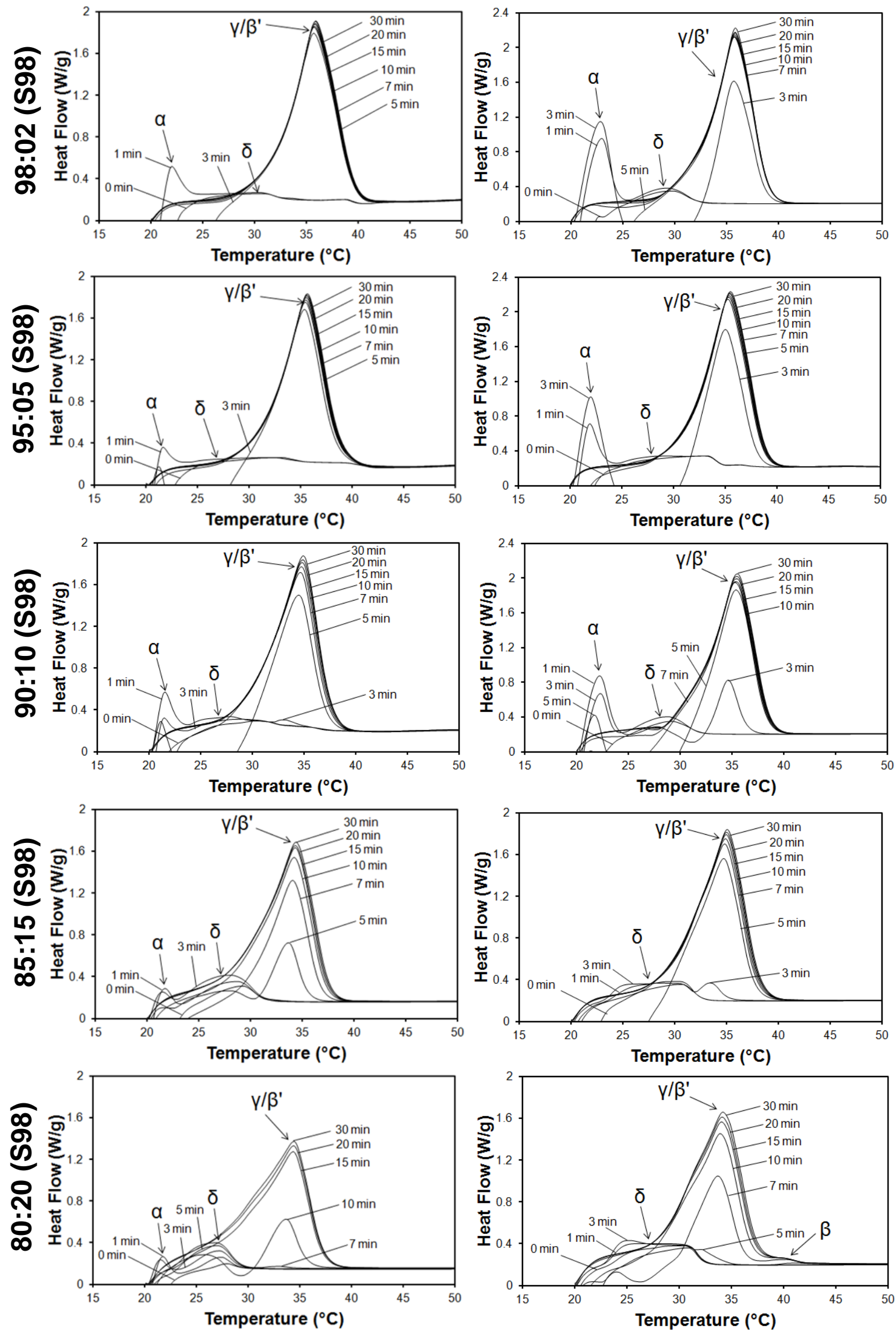

(A)

(B)

Figure 2 

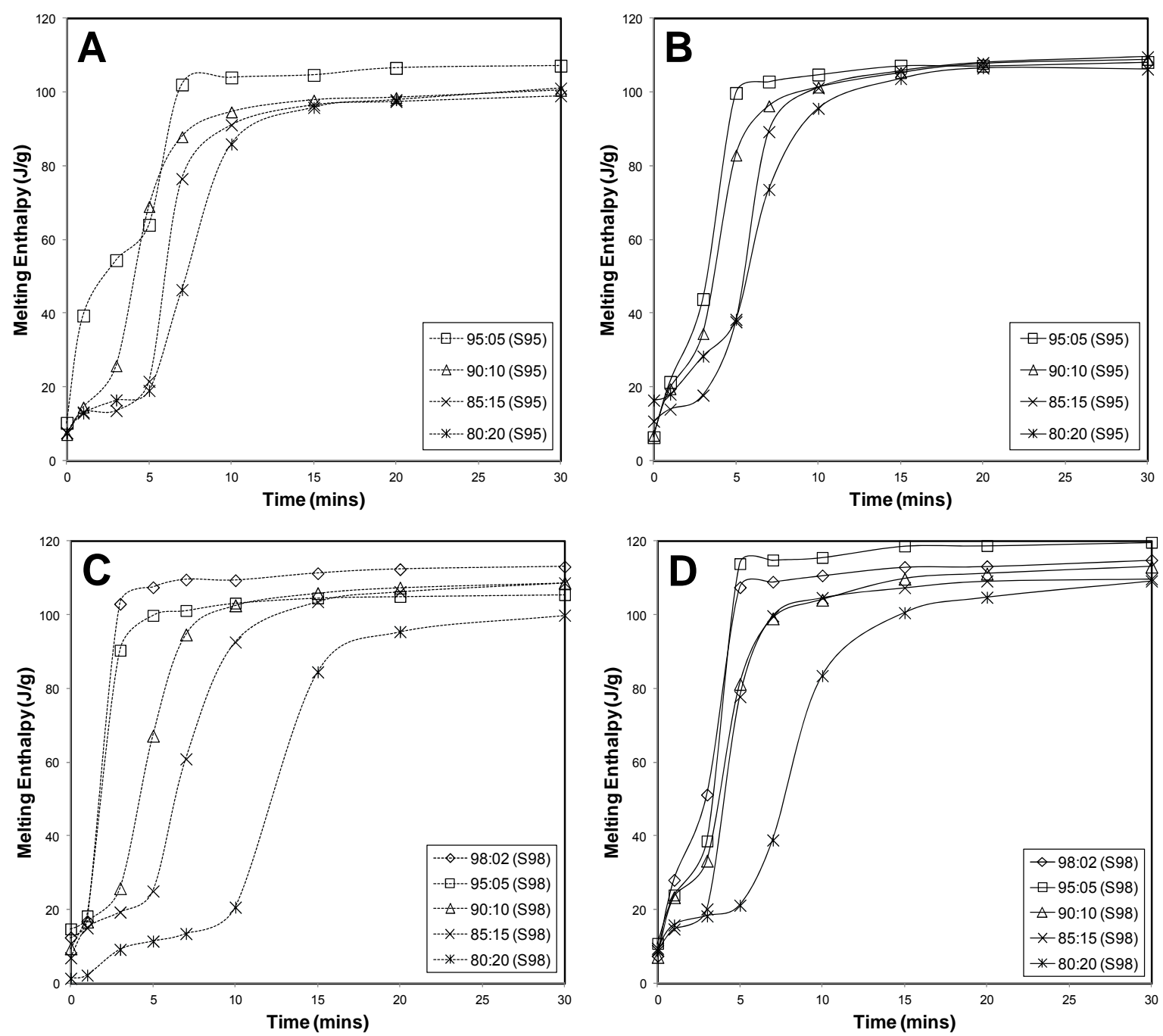

Figure 3 

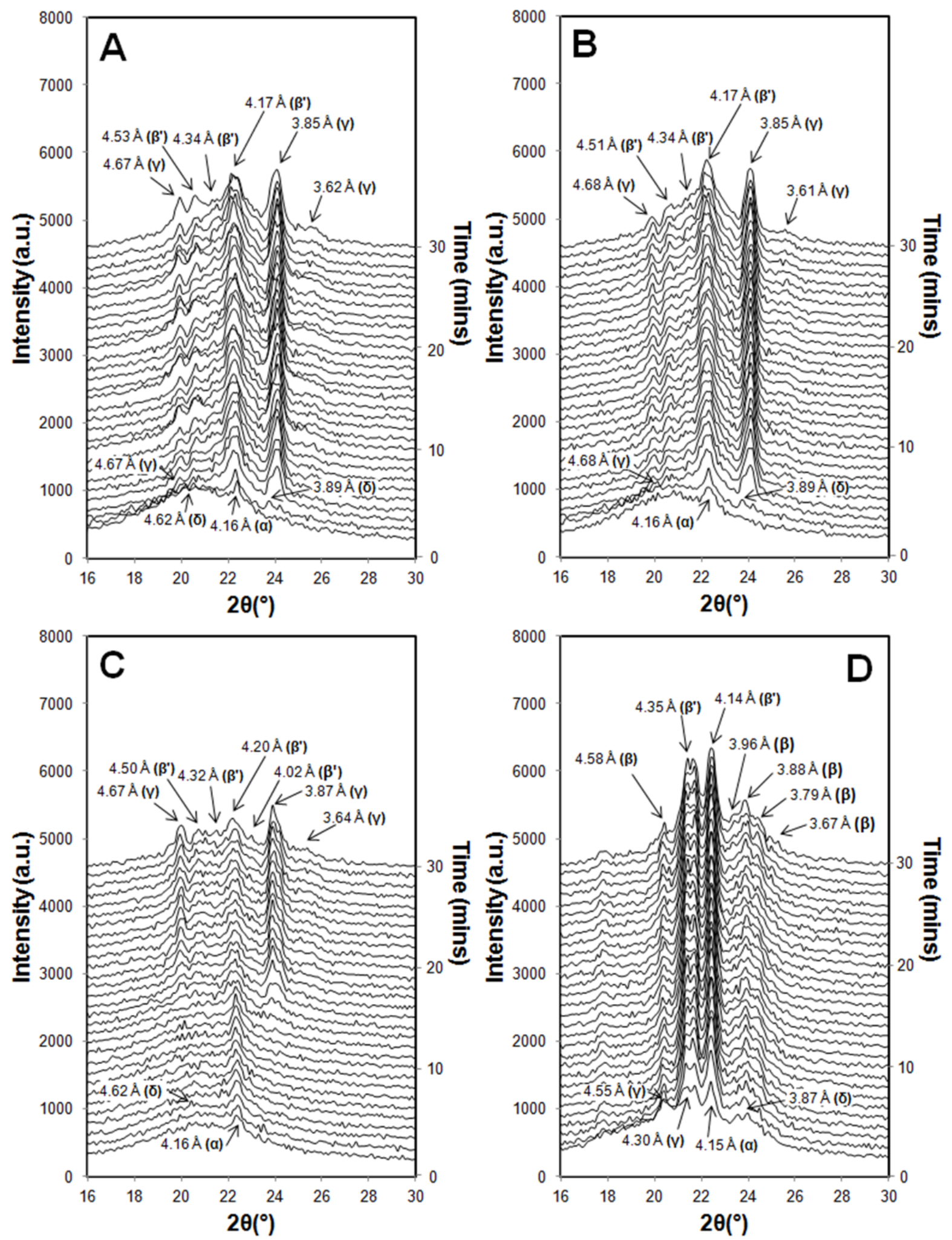

Figure 4 


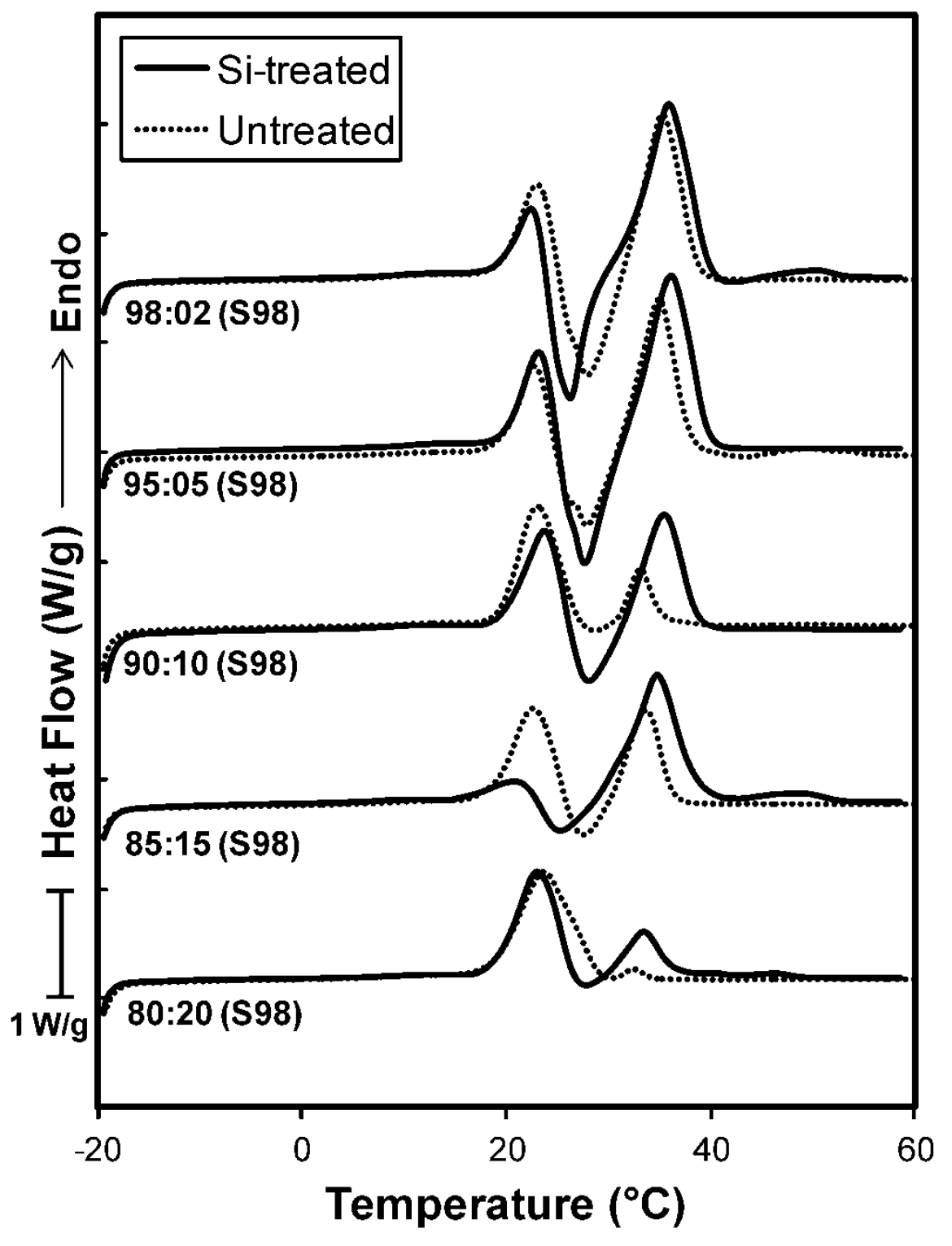

Figure 5 

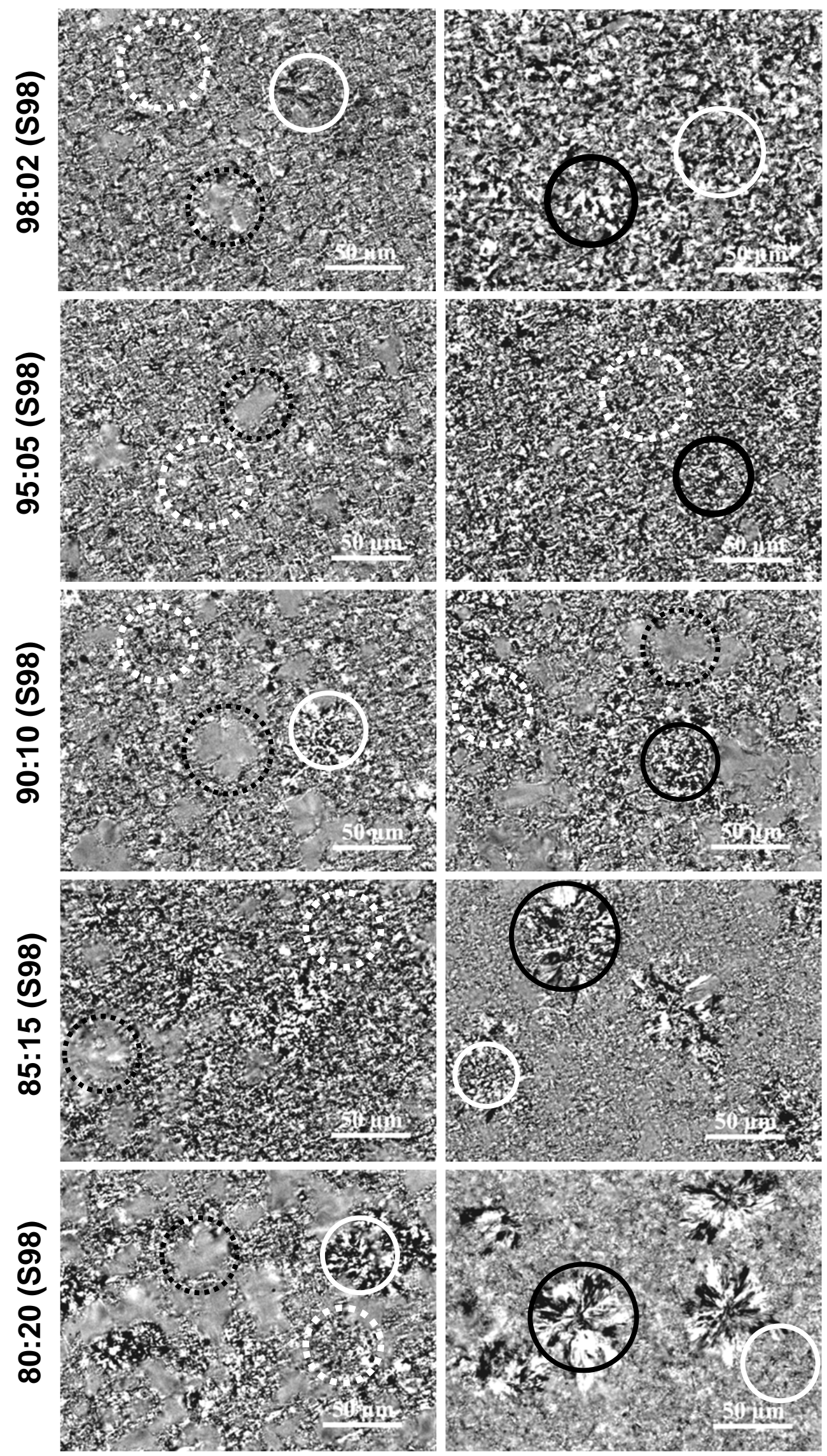

(A)

(B)

Figure 6 

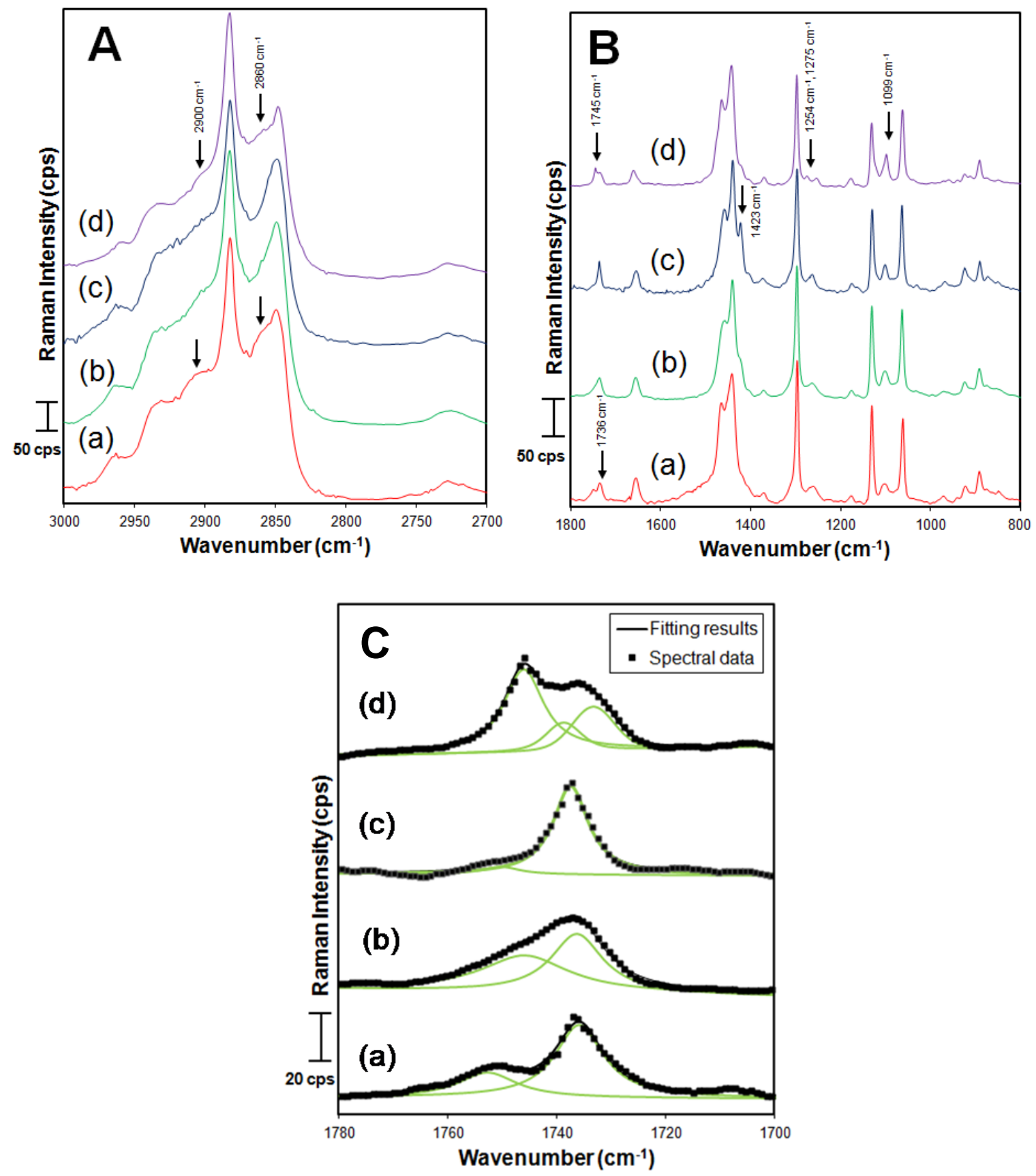

Figure 7 

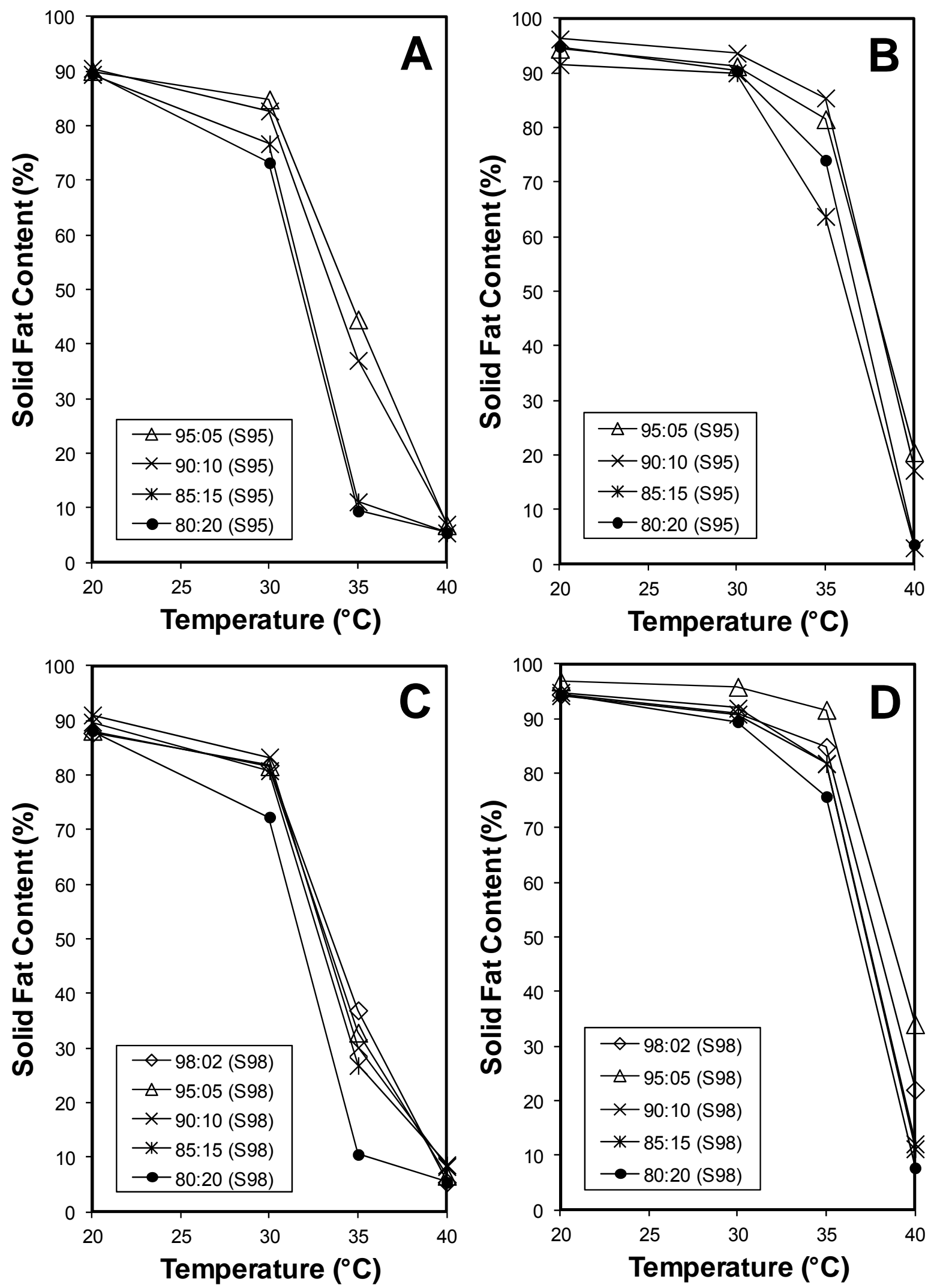

Figure 8 


\section{List of Figures in Supporting Information}

Figure S1. Melting profiles indicating polymorphic forms $\left(\alpha, \delta, \gamma\right.$ and $\left.\gamma^{+} \beta^{\prime}\right)$ of $(A)$ untreated and (B) Si-treated S95 stearins obtained by the stop-and-return DSC method in which the isothermal crystallisation at $20^{\circ} \mathrm{C}$ was interrupted after $1,2,3,5,7,10,15,20$ and 30 minutes.

Figure S2. Non-isothermal DSC heating thermograms of untreated and Si-treated (A) S95 and (B) S98 stearins. Melting exotherms were obtained by heating the samples to $60^{\circ} \mathrm{C}$ at $5^{\circ} \mathrm{C} / \mathrm{min}$ minutes after previously cooling from $80^{\circ} \mathrm{C}$ to $-20^{\circ} \mathrm{C}$ at $5^{\circ} \mathrm{C} / \mathrm{min}$.

Figure S3. Microstructure of (A) untreated and (B) Si-treated S95 stearins acquired under polarised light after crystallising at $20^{\circ} \mathrm{C}$ for 7 days. Different polymorphic forms (identified by Raman microscopy) are indicated by circles outlined on the microstructure. The white dashed circle represents the $\gamma$ form, white solid circle represents the $\gamma^{+} \beta^{\prime}$ form, black dashed circle represents the $\beta$ ' form and black solid circle represents the $\beta$ form. 

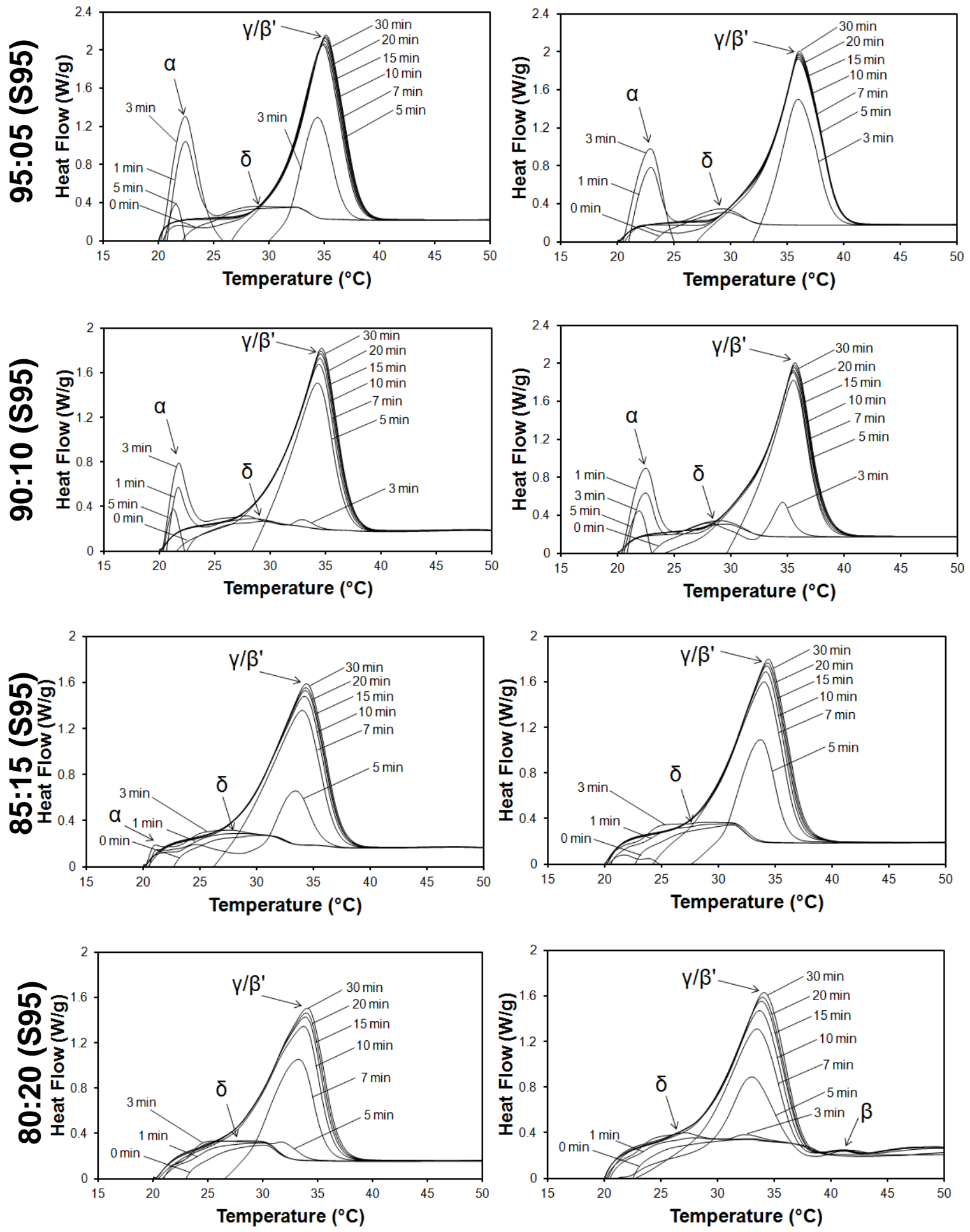

(A)

(B)

Figure S1 


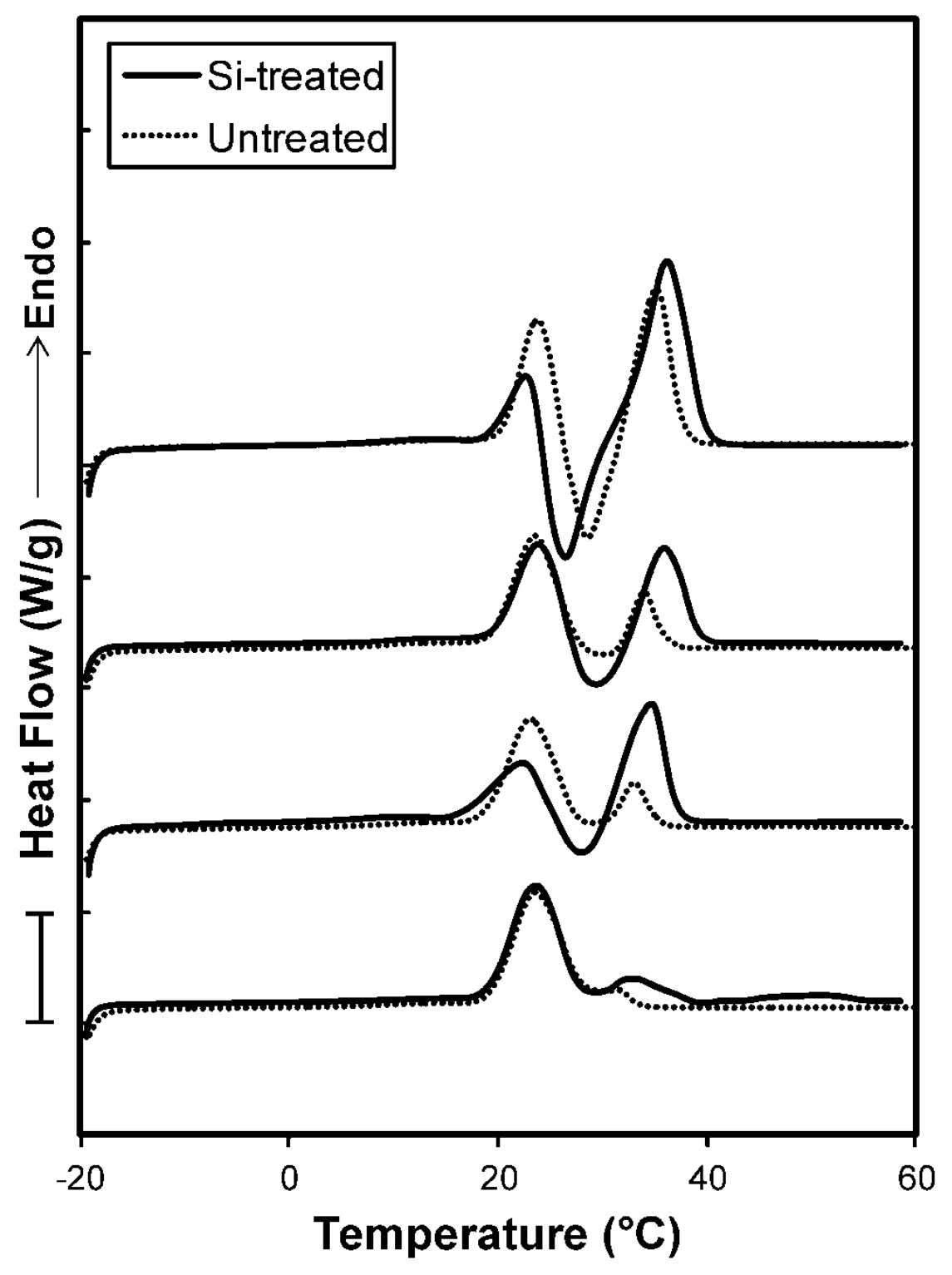

Figure S2 

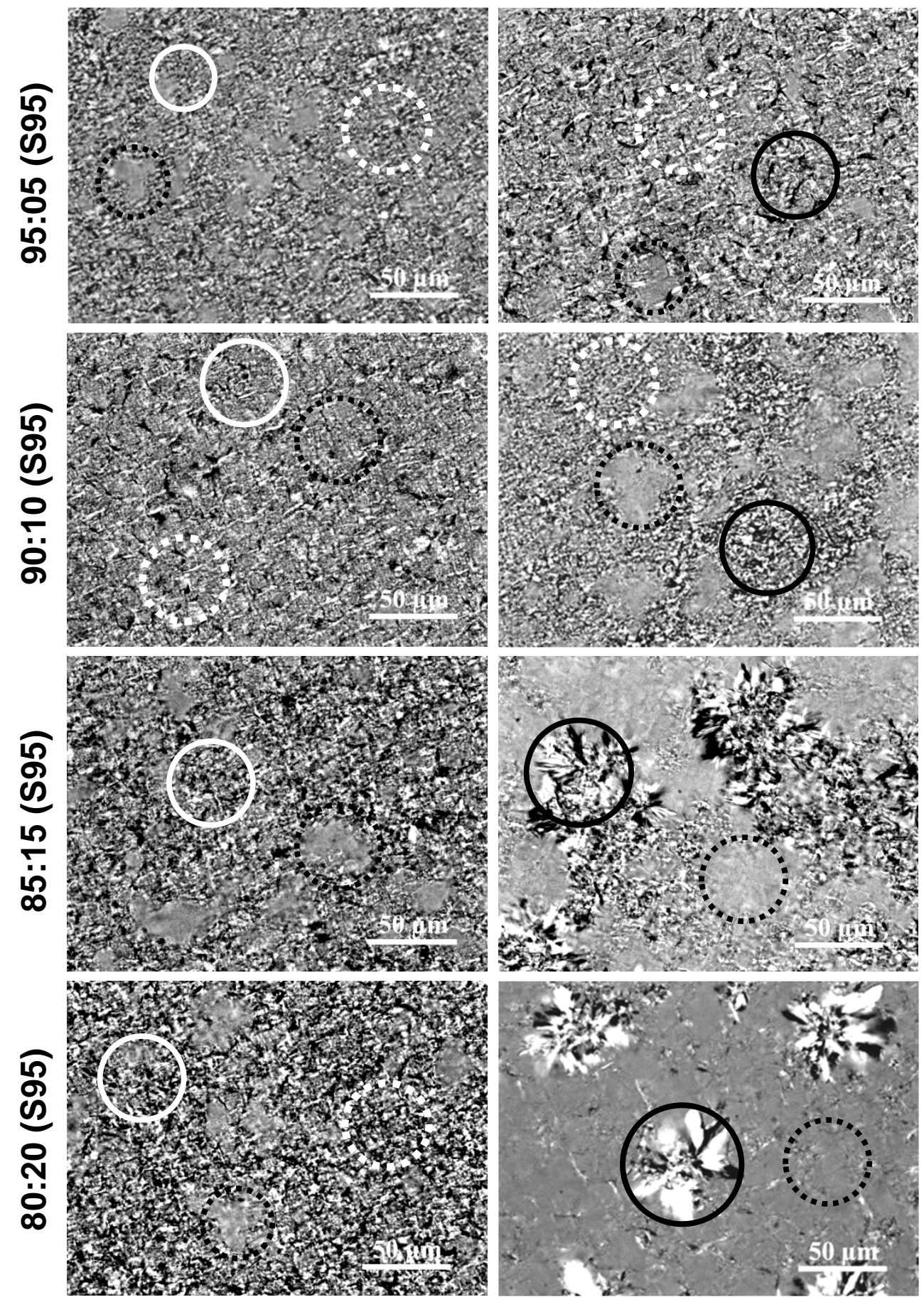

(A)

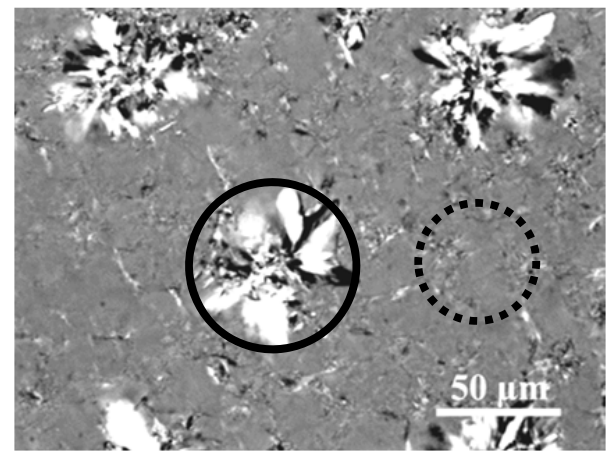

(B)

Figure S3 